\title{
Magnetic reconnection as an element of turbulence
}

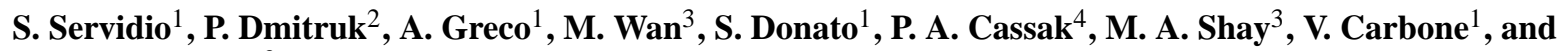 \\ W. H. Matthaeus ${ }^{3}$ \\ ${ }^{1}$ Dipartimento di Fisica, Università della Calabria, 87036 Cosenza, Italy \\ ${ }^{2}$ Departmento de Física, Facultad de Ciencias Exactas y Naturales, Universidad de Buenos Aires, and IFIBA, CONICET, \\ Ciudad Universitaria, 1428, Buenos Aires, Argentina \\ ${ }^{3}$ Bartol Research Institute and Department of Physics and Astronomy, University of Delaware, Newark, DE 19716, USA \\ ${ }^{4}$ Department of Physics, West Virginia University, Morgantown, WV 26506, USA
}

Received: 30 July 2011 - Revised: 13 September 2011 - Accepted: 14 September 2011 - Published: 12 October 2011

\begin{abstract}
In this work, recent advances on the study of reconnection in turbulence are reviewed. Using direct numerical simulations of decaying incompressible two-dimensional magnetohydrodynamics (MHD), it was found that in fully developed turbulence complex processes of reconnection locally occur (Servidio et al., 2009, 2010a). In this complex scenario, reconnection is spontaneous but locally driven by the fields, with the boundary conditions provided by the turbulence. Matching classical turbulence analysis with a generalized Sweet-Parker theory, the statistical features of these multiple-reconnection events have been identified. A discussion on the accuracy of our algorithms is provided, highlighting the necessity of adequate spatial resolution. Applications to the study of solar wind discontinuities are reviewed, comparing simulations to spacecraft observations. New results are shown, studying the time evolution of these local reconnection events. A preliminary study on the comparison between MHD and Hall MHD is reported. Our new approach to the study of reconnection as an element of turbulence has broad applications to space plasmas, shedding a new light on the study of magnetic reconnection in nature.
\end{abstract}

\section{Introduction}

Magnetic reconnection is a process that occurs in many astrophysical and laboratory plasmas (Sonnerup, 1970; Vasyliunas, 1975; Moffatt, 1978). Systems like the solar surface (Parker, 1983), the magnetosphere (Sonnerup et al., 1981; Paschmann, 2008), the solar wind (Gosling and Szabo, 2008), the magnetosheath (Retinò et al., 2007; Sundkvist et al., 2007), and laboratory plasmas (Taylor, 1986; Yamada, 2007; Brown et al., 2006) represent just some of the classical

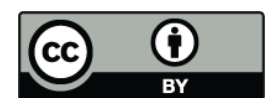

Correspondence to: S. Servidio (sergio.servidio@fis.unical.it) systems in which magnetic reconnection occurs. Another underlying common feature of the above systems is the presence of turbulence (Bruno and Carbone, 2005), so a simultaneous description of both reconnection and turbulence is needed.

In the past $60 \mathrm{yr}$, most of the theoretical effort has been addressed to the study of the basic physics of reconnection, concentrating on idealized geometries. Generally, these twodimensional (2-D) models are characterized by a strong current density peak, where a magnetic $X$-type neutral point is found (Dungey, 1958). A well-known description of this process was provided by Sweet (Sweet, 1958) and Parker (Parker, 1957). In their work, employing conservation of mass, pressure balance and constancy of the electric field, the essential large scale dynamics of magnetic reconnection was described. In this configuration, a narrow layer called the "diffusion region" forms, and here the field-lines break and reconnect. This process produces a plasma flow into the layer, accompanied by an outflow along the neutral sheet.

Reconnection may be self-sustaining with ad-hoc boundary conditions that supply flux, or it may continue until available magnetic flux is exhausted (Sato et al., 1978; Birn et al., 2001). In many cases the reconnecting system has been idealized as occurring in a limited spatial region, employing a "rigid-box" topology in which the magnetic field is often arbitrarily chosen to be straight at the inflow-side boundaries. Moreover, simplified "outflow" boundaries are employed. However, such idealized conditions rarely occur in nature, since plasmas may frequently experience turbulence (Bruno and Carbone, 2005; Retinò et al., 2007; Sundkvist et al., 2007). In turbulence, magnetic reconnection may behave in a less predictable way, departing considerably from rigidbox models.

We view reconnection as an element of turbulence itself: it would be difficult to envision a turbulent cascade that proceeds without change of magnetic topology. Furthermore, turbulence provides a natural boundary condition, as opposed

Published by Copernicus Publications on behalf of the European Geosciences Union and the American Geophysical Union. 
to arbitrary (imposed) conditions. Although some suggestions have been made regarding both the general role of reconnection in magnetohydrodynamic (MHD) turbulence (Matthaeus and Lamkin, 1986; Strauss, 1980; Carbone et al., 1990; Dmitruk and Matthaeus, 2006; Veltri, 1999; Rappazzo et al., 2007) and the impact of small scale turbulence on reconnection of large structures (Matthaeus and Montgomery, 1980; Matthaeus and Lamkin, 1986; Malara et al., 1992; Lazarian and Vishniac, 1999; Lapenta, 2008; Veltri, 1999), only recently a quantitative study of reconnection in turbulence has been presented (Servidio et al., 2009, 2010a). In the scenario proposed by Servidio et al., multiple-reconnection events are present in turbulence. The properties of these events depend on the local topology of the magnetic field and the local turbulence conditions.

Simulating numerically the problem of reconnection involves some subtle issues: scientists, generally tempted by the "high-Reynolds-number-dream", may push too much on the resolution, neglecting that this is strongly limited by the imposed dissipation (or vice-versa). Recently, Wan et al. (2010) demonstrated that an insufficient numerical (grid) resolution may strongly damage the small scale properties of both turbulence and reconnection. Here we summarize these main results, providing suggestions and numerical recipes in order to correctly simulate the problem of reconnection in turbulence.

Our ideas on magnetic reconnection have broad applications, and one of them is the turbulent solar wind. In the free solar wind, in fact, strong magnetic discontinuities are commonly observed (Burlaga, 1968; Tsurutani and Smith, 1979; Ness and Burlaga, 2001; Neugebauer, 2006). These consist of rapid changes of the magnetic field, across narrow layers. It is natural to ask whether these discontinuities are related to the process of reconnection. In recent works by Greco et al. $(2008,2009)$ a link between these rapid changes of the magnetic field and the presence of intermittent current sheets was proposed. In the present paper we retrace these ideas providing evidence that reconnection and discontinuities may be different faces of the same coin.

Although the combined effects of turbulence and reconnection are likely to be important in a variety of physical systems, the investigations described here are carried out in the limited context of incompressible MHD, for which the turbulence problem, as well as the well-resolved reconnection problem, are already very demanding. We recognize that effects not included here, such as fully kinetic plasma dynamics (Drake et al., 2006; Fu et al., 2006; Huang et al., 2010; Daughton et al., 2011) and strongly compressive effects (Webb et al., 2003; Jokipii and Lee, 2010) can have important influences on turbulence and reconnection. Especially significant are influences on particle acceleration which can only be addressed in the present class of models using test particles (Matthaeus et al., 1984; Dmitruk et al., 2003). We proceed employing a more limited set of models with the goal of identifying the basic physical principles
Table 1. Table of runs. The second column indicates the resolution of the simulation, the third column the Reynolds number. In the fourth column, the energy shell initially excited is reported. The last column shows the time $t^{*}$ at which the analysis have been performed.

\begin{tabular}{lrrrr}
\hline & Resolution & $\begin{array}{r}R_{\mu} \\
\left(=R_{\nu}\right)\end{array}$ & $\begin{array}{r}\text { Fourier modes } \\
\text { at } t=0\end{array}$ & $t^{*}$ \\
\hline Run 1 & $4096^{2}$ & 1700 & $4 \leq k \leq 10$ & 0.4 \\
Run 2 & $4096^{2}$ & 2500 & $5 \leq k \leq 30$ & 0.2 \\
Run 3 & $4096^{2}$ & 2500 & $3 \leq k \leq 10$ & 0.7 \\
Run 4 & $8192^{2}$ & 5000 & $5 \leq k \leq 30$ & 0.3 \\
Run 5 & $8192^{2}$ & 5000 & $8 \leq k \leq 20$ & 0.3 \\
Run 6 & $16384^{2}$ & 10000 & $5 \leq k \leq 30$ & 0.3 \\
\hline
\end{tabular}

at work in the turbulence-reconnection dynamical system. Indeed this may be the only tractable approach available at present to undertake this difficult nonlinear problem. In the future, more complete models may be able to address some or all of the basic physics that are attempting to describe.

In this review paper new results will also be presented. In particular, we will analyze the processes of reconnection during turbulent relaxation, following in time the dynamics of 2-D MHD turbulence. At different times, we will perform our analysis characterizing the statistical properties of this complex dynamical system. MHD theory, however, lacks important plasma length-scales. The first step-forward in this regard is to use models such as Hall magnetoydrodynamics (Hall MHD). Dispersive Hall MHD models can, in fact, capture some of the physics of the ion skin depth and therefore describe phenomena that are commonly observed in plasmas (Roberts and Taylor, 1962; Matthaeus et al., 2008). In this work, a preliminary comparison between results of MHD and Hall MHD will be presented.

The outline of the paper is as follows: in Sect. 2, an overview on the main features of 2-D MHD turbulence will be presented. The methodology and the statistical analysis of reconnection in turbulence will be reviewed in Sect. 3, establishing a link between length-scales in turbulence and in reconnection, and presenting a generalized Sweet-Parker theory. In Sect. 4 the time dynamics of reconnection in turbulence will presented, while preliminary results of Hall MHD will be shown in Sect. 5. The importance of numerical accuracy will be emphasized in Sect. 6. Possible applications of all the above ideas to the turbulent solar wind will be reviewed in Sect. 7 while, in the last Section, conclusions will be discussed.

\section{Overview on 2-D MHD turbulence}

The 2-D incompressible MHD equations can be written in terms of the magnetic potential $a(x, y)$ and the stream function $\psi(x, y)$. By choosing a uniform mass density $\rho=1$, the 


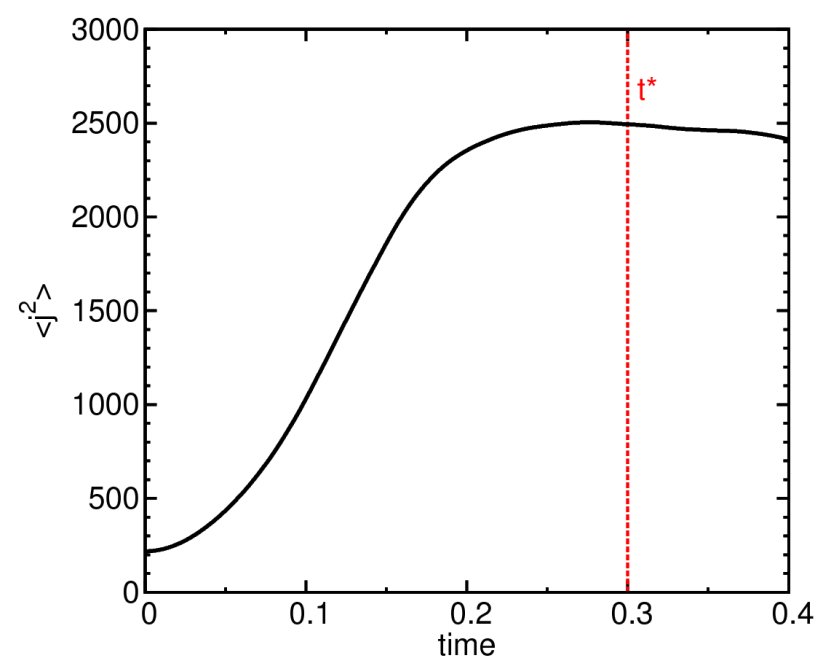

Fig. 1. (Color online) The current density $\left\langle j^{2}\right\rangle$ as a function of time. The peak is reached around $t^{*}=0.3$ (vertical red dashed line). Results are shown for Run 4, and other runs show similar timebehavior (see Table 1 for more details).

equations read (Biskamp, 1993):

$$
\frac{\partial \omega}{\partial t}=-(\boldsymbol{v} \cdot \nabla) \omega+(\boldsymbol{b} \cdot \nabla) j+R_{\nu}^{-1} \nabla^{2} \omega
$$

$\frac{\partial a}{\partial t}=-(\boldsymbol{v} \cdot \nabla) a+R_{\mu}^{-1} \nabla^{2} a$,

where the magnetic field is $\boldsymbol{b}=\nabla a \times \hat{z}$, the velocity $\boldsymbol{v}=$ $\nabla \psi \times \hat{z}$, the current density $j=-\nabla^{2} a$, and the vorticity $\omega=-\nabla^{2} \psi$. Equations (1)-(2) are written in Alfvén units (Matthaeus and Montgomery, 1980) with lengths scaled to $L_{0}$. The latter is a typical large scale length such that the box size is set to $2 \pi L_{0}$. Velocities and magnetic fields are normalized to the root mean square Alfvén speed $V_{\mathrm{A}}$ and time is scaled to $L_{0} / V_{\mathrm{A}} . R_{\mu}$ and $R_{\nu}$ are the magnetic and kinetic Reynolds numbers, respectively (at scale $L_{0}$.) The latter coefficients are reciprocals of kinematic viscosity and resistivity.

Equations (1)-(2) are solved in a periodic Cartesian geometry $(x, y)$, using a well tested dealiased (2/3 rule) pseudospectral code (Ghosh et al., 1993). We employ a standard Laplacian dissipation term with constant dissipation coefficients. The latter are chosen to achieve both high Reynolds numbers and to ensure adequate spatial resolution. A detailed discussion of these issues has been given by Wan et al. (2010), and will be reviewed here in Sect. 6. We report on runs with resolution from $4096^{2}$ up to $16384^{2}$ grid points, reaching Reynolds numbers $R_{\nu}=R_{\mu} \sim 10000$. Time integration is second order Runge-Kutta and double precision is employed. A description of all the runs is reported in Table 1.

Considering a representation of the fields in the Fourier space, for a particular run (in this case Run 4, cf. Table 1),

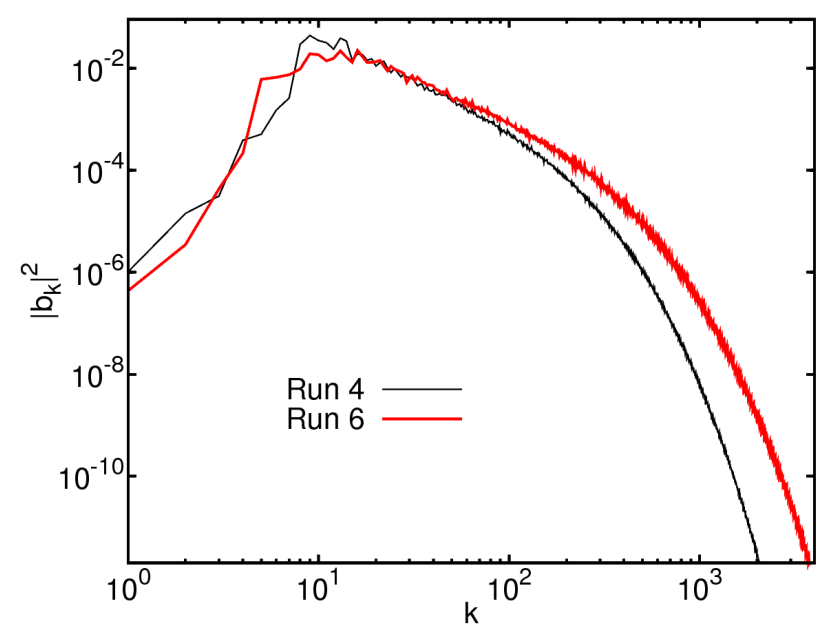

Fig. 2. (Color online) Power spectra of magnetic field for two different simulations: Run 4 (8192 ${ }^{2}$, thin-black) and Run $6\left(16384^{2}\right.$, thick-red). As expected, higher resolution simulations have wider inertial range.

the energy is initially concentrated in the shell $5 \leq k \leq 30$ (wavenumber $k$ in units of $1 / L_{0}$ ), with mean value $E=$ $\frac{1}{2}\left\langle|\boldsymbol{v}|^{2}+|\boldsymbol{b}|^{2}\right\rangle \simeq 1,\langle\ldots\rangle$ denoting a spatial average. Random uncorrelated phases are employed for the initial Fourier coefficients. The latter implies that the cross helicity, defined as $H_{c}=1 / 2\langle\boldsymbol{v} \cdot \boldsymbol{b}\rangle$, is negligible. The kinetic and the magnetic energy at the beginning of the simulation are chosen to be equal.

To compare different runs, we consider for the statistical analysis the state of the system at which the mean square current density $\left\langle j^{2}\right\rangle$ is very near to its peak value (see Fig. 1). At this instant of time the peak of small scale turbulent activity is achieved. Since the system is homogeneous and isotropic, it is useful to compute the omnidirectional power spectra, reported in Fig. 2. In this figure, a comparison between Run 4 (moderate resolution) and Run 6 (high resolution) is shown. The power spectrum manifests a broad inertial range, typical of turbulence.

When turbulence is fully developed, coherent structures appear. They can be identified as magnetic islands (or vortices). A typical complex pattern of 2-D MHD turbulence is shown in Fig. 3, at high Reynolds numbers (Run 6). In the figure is represented a contour plot of the current $j$, together with the in-plane magnetic field (line contour of $a$ ). The current density $j$ becomes very high in narrow layers between islands.

In Fig. 4a, a zoom into the turbulent field is represented, showing that the current is bursty in space. This behavior of the current is related to the intermittent nature of the magnetic field (Sorriso-Valvo et al., 1999; Mininni and Pouquet, 2009) and can be interpreted as a consequence of fast and local relaxation processes (Servidio et al., 2008; Greco et al., 2008). As reported in Fig. 4b, the out of plane component of 


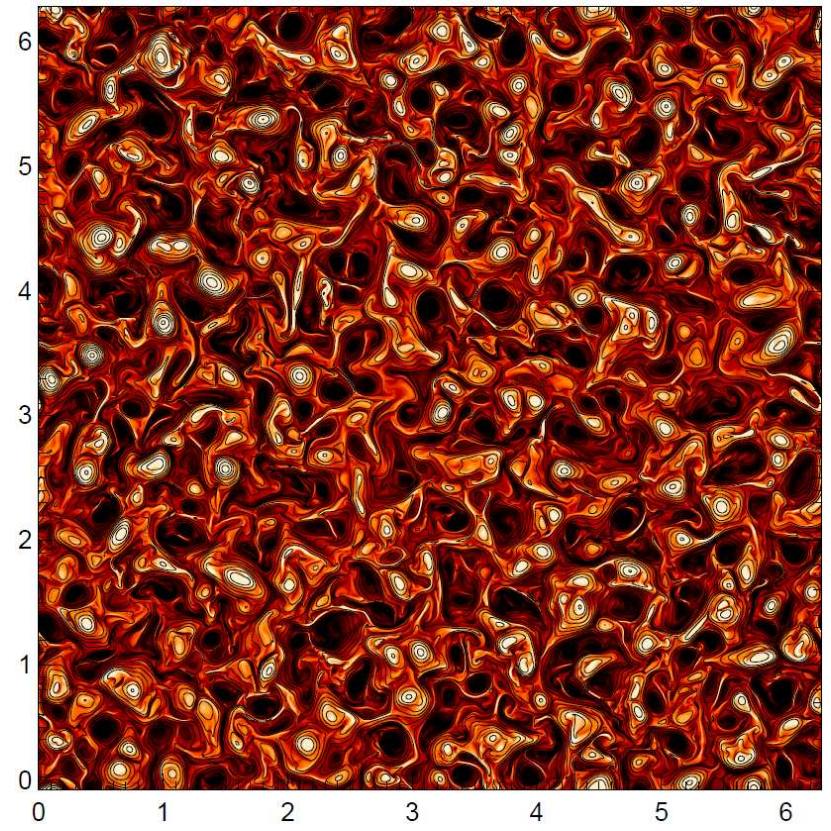

Fig. 3. (Color online) Shaded contour of the current density $j$ together with the line contours of the magnetic potential $a$ (Run 6 , at $\left.t^{*}=0.3\right)$.

the magnetic potential $a$ shows a complex pattern of multiscale islands, having a wide distribution of sizes. In between these structures the current can be very high. The probability distribution function (PDF) of the current density strongly departs from a Gaussian, as shown in Fig. 4c. These coherent structures interact non-linearly, merge, stretch, connect, attract and repulse each other. Reconnection is a major element of this complex interaction.

\section{Local reconnection events in turbulence}

The reconnection rate of two islands is given by the electric field at the $X$-point. This is related to the fact that the magnetic flux in a closed 2-D island is computed as the integrated magnetic field normal to any contour connecting the central $O$-point (maximum or minimum of $a$ ) with any other specified point. Choosing that point to be an $X$-point bounding the island, we find that the flux in the island is just $a(O-$ point $)--a(X-$ point $)$ (Smith et al., 2004). Flux is always lost at the $O$-point in a dissipative system, so the time rate of change of the flux due to activity at the $X$-point is

$$
\frac{\partial a}{\partial t}=-E_{\times}=\left(R_{\mu}^{-1} j\right)_{\times},
$$

where $E_{\times}$is an abbreviation for the electric field measured at the $X$-point (analogously for the current $j_{\times}$). Eq. (3) follows from the Ohm's law
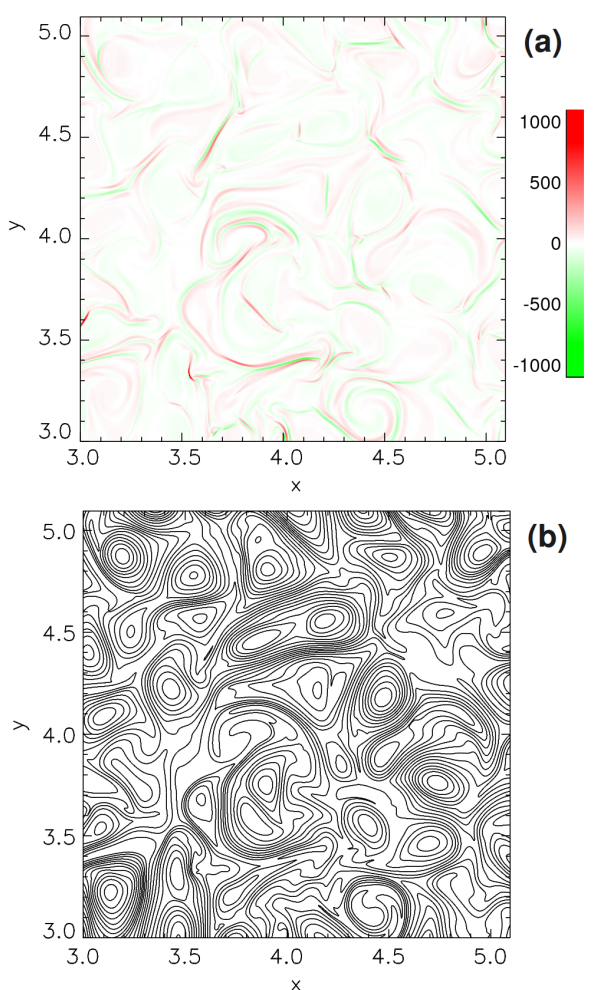

(b)

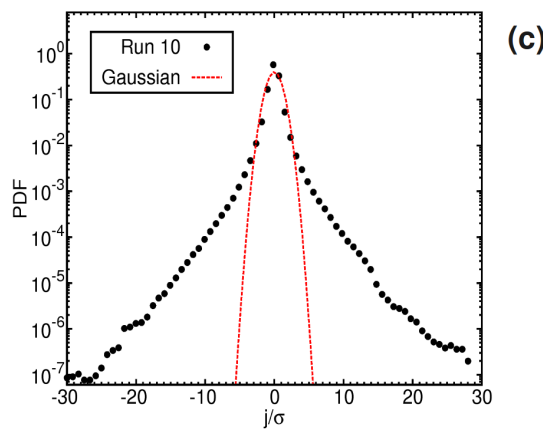

Fig. 4. (Color online) (a) Shaded-contour of the current density $j$ in a sub-region of the simulation box, showing that, in between magnetic islands, $j$ is high; (b) Line-contour of the in-plane magnetic field (iso-lines of the magnetic potential $a$ ); (c) PDF of the current density, normalized to its variance, for high Reynolds number turbulence (black bullets). The Gaussian distribution is reported for comparison (red-dashed line).

$\boldsymbol{E}=-\boldsymbol{v} \times \boldsymbol{b}+R_{\mu}^{-1} \mathbf{j}$,

which in 2-D involves only the out of plane component $E_{z}=-(\boldsymbol{v} \times \boldsymbol{b})_{z}+R_{\mu}^{-1} j$. Therefore, in order to describe the local processes of reconnection that spontaneously develop in turbulence we examine the topology of the magnetic potential studying the Hessian matrix of a (Rana, 2004), defined as

$H_{i, j}^{a}(\boldsymbol{x})=\frac{\partial^{2} a}{\partial x_{i} \partial x_{j}}$, 


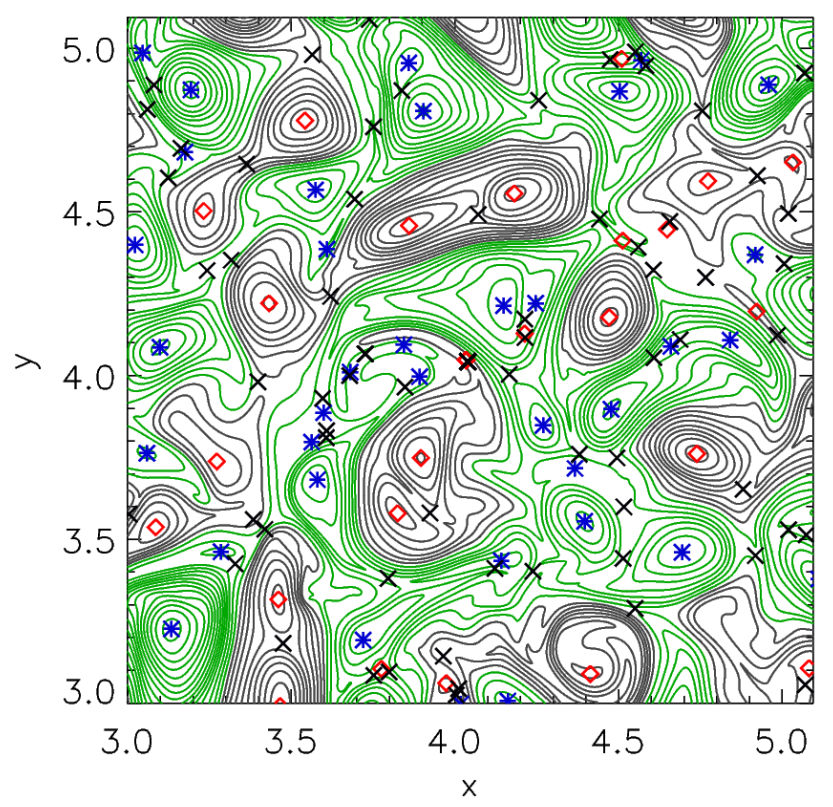

Fig. 5. (Color online) Contour plot of the magnetic potential $a$ with the position of all the critical points: $O$-points (blue stars for the maxima and red open-diamonds for the minima) and $X$-points (black $x$ ).

which we evaluate at the neutral points of the magnetic field. Further details on the methodology are provided in Servidio et al. (2010a). Here we briefly summarize the main steps of the analysis:

1. Identify critical points at $\boldsymbol{x}^{*}$, where $\nabla a=0$.

2. Compute the Hessian matrix, given by Eq. (5), at $\boldsymbol{x}^{*}$.

3. Compute eigenvalues $\lambda_{1}$ and $\lambda_{2}$ of $H_{i, j}^{a}\left(x^{*}\right)$, with $\lambda_{1}>$ $\lambda_{2}$.

4. Classify the critical point as maximum (both $\lambda_{i}<0$ ), minimum (both $\lambda_{i}>0$ ) and saddle points (or $X$-points) $\left(\lambda_{1} \lambda_{2}<0\right)$.

5. Compute eigenvectors at each $X$-point. The associated unit eigenvectors are $\hat{e}_{s}$ and $\hat{e}_{l}$, where coordinate $s$ is associated with the minimum thickness $\delta$ of the current sheet, while $l$ is associated with the elongation $\ell$. Note that the local geometry of the diffusion region near each $X$-point is related to the Hessian eigenvalues $\lambda_{1}=\frac{\partial^{2} a}{\partial s^{2}}$ and $\lambda_{2}=\frac{\partial^{2} a}{\partial l^{2}}$.

6. According to Eq. (3), the reconnection rates are given by the electric field at the $X$-points. These rates are then normalized to the mean square fluctuation $\delta b_{\mathrm{s}}^{2}$, appropriate for Alfvènic turbulence.

In Fig. 5 we show the magnetic potential with the critical point locations, obtained with the above procedure. In this

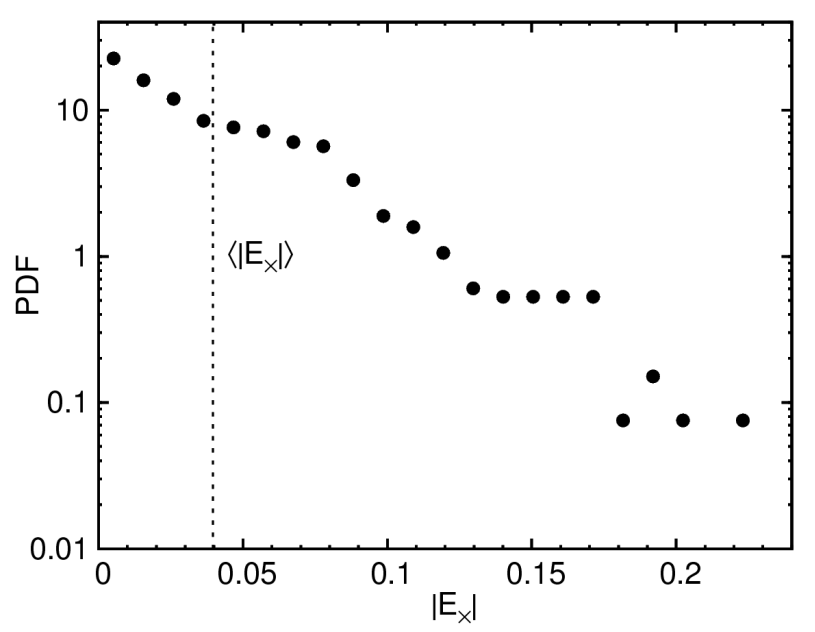

Fig. 6. (Color online) Probability distribution function of reconnection rates in turbulence (lin-log scale). Vertical dotted line represents the mean value of the distribution.

complex picture the $X$-points link islands with different size and energy.

The distribution of reconnection rates $\operatorname{PDF}\left(\left|E_{\times}\right|\right)$is reported in Fig. 6 for Run 4. Different simulations give qualitatively same results. The PDF of the electric field at the $X$ points is broad and peaked around zero value. For this particular simulation (Run 4), the mean value of the reconnection rate is $\simeq 0.04$, with strong variations from the average, that is values are found in the range $\left|E_{\times}\right| \in\left[10^{-6}, 0.3\right]$. In terms of the global parameters, this observed range of reconnection rates varies from very slow to fast. In this sense the typical reconnection rate in turbulence is found to be far higher than what is expected based on a simple global application of the Sweet-Parker rate $E_{\times} \sim R_{\mu}^{-1 / 2}$. We now examine more details of how these rates arise.

From a scaling analysis

$\frac{\ell}{\delta} \simeq \sqrt{\lambda_{R}}$

where $\lambda_{R}=\left|\frac{\lambda_{1}}{\lambda_{2}}\right|$. In the case in which the reconnection is in a stationary state, the rate depends on the above aspect ratio $\lambda_{R}$, satisfying the scaling

$E_{\times} \sim \frac{\ell}{\delta} \sim \sqrt{\lambda_{R}}$.

In Fig. 7, a scatter plot of the reconnection rates against the aspect ratio $\lambda_{R}$ is shown. There is a clear trend in this figure, showing that Eq. (7) is satisfied. This suggests that locally the reconnection processes depend on the geometry and that they therefore are in a quasi steady-state regime.

The approximate power-law scaling seen in Fig. 7 at larger values of $\lambda_{R}$ suggests that Eq. (7) holds for the fastest reconnection events. The weaker reconnection events evidently 


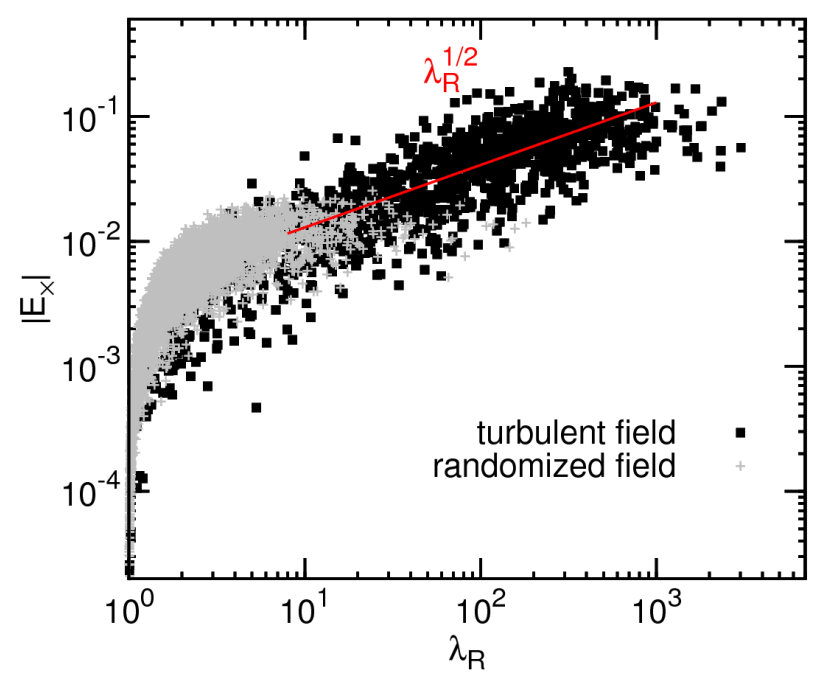

Fig. 7. (Color online) Scatter plot (black full squares) of the reconnection rates vs. the geometry of the reconnection region (ratio of the eigenvalues $\lambda_{R}$ ). The presence of a power-law fit (red line) demonstrates that there is a relation between the reconnection rate and the geometry of the diffusion region. The relative "randomized" reconnection rate is reported with (gray) crosses.

follow a different scaling. We now show that the collection of slowly reconnecting (or even non-reconnecting) $X$ point regions is associated with a distribution of magnetic fields that is Gaussian. As described by Servidio et al. (2009, 2010a), we now employ a phase-randomizing procedure: the original turbulent field is compared with a hybrid field that has the same spectrum but random phases. The coherency of a turbulent pattern is, in fact, hidden in the phases of the Fourier expansion. Using this technique, one can distinguish between slow (Gaussian) and fast (non-Gaussian) reconnection events (Servidio et al., 2010a). As it can be seen from Fig. 7, the reconnection rates of the incoherent randomized magnetic field are on average much weaker than for the original case and they do not manifest any dependence on the aspect ratio of the eigenvalues. In fact the part of the distribution where we found the strongest reconnection sites and the scaling relation with aspect ratio is completely absent in the Gaussianized case. We would like to stress that phasecoherency analysis are widely used in the literature, and they are generally adopted to identify coherent structures (Hada et al., 2003; Koga and Hada, 2003; Koga et al., 2007; Sahraoui and Goldstein, 2010).

We remark on our procedures for verification, as we have found that the stable and accurate determination of the distribution of reconnection rates requires considerable care, especially with regard to spatial resolution. Numerous test cases were examined in this regard. The numerical results shown were verified in a number of ways: We compared runs with different time steps and spatial resolutions, checked that the
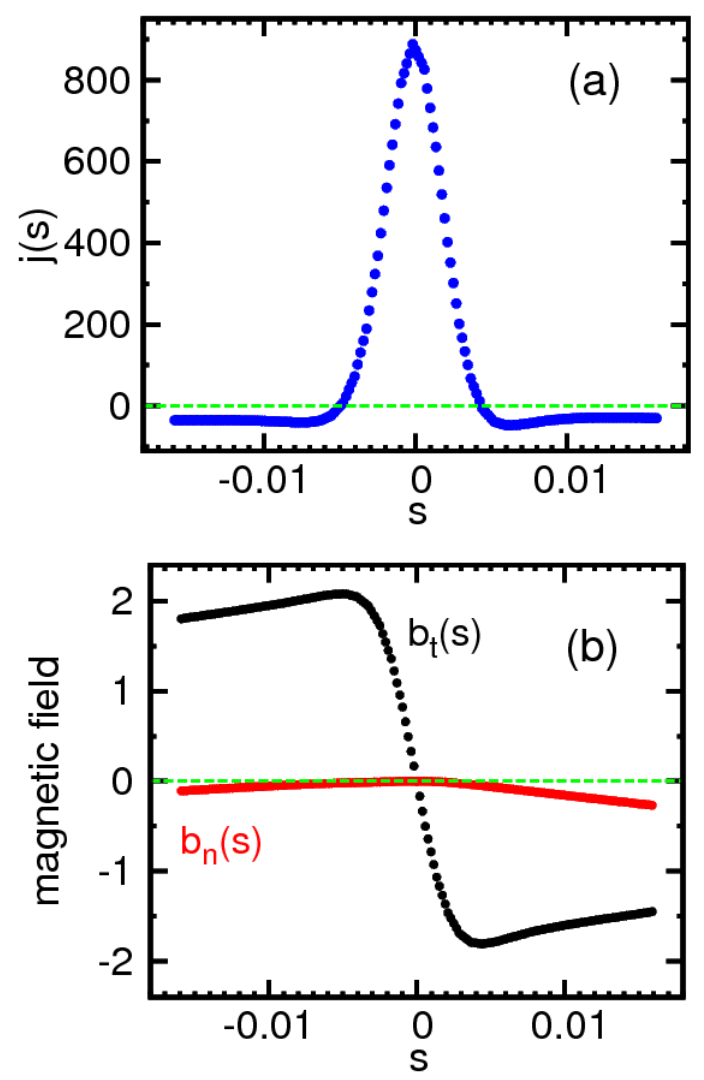

Fig. 8. (Color online) Profiles of the current density (a) in the vicinity of an $X$-point (blue bullets). The $X$-point is located at $s=0$. In panel (b) the tangential $\left(b_{\mathrm{t}}\right.$, black) and the normal $\left(b_{\mathrm{n}}\right.$, red) components of the magnetic field are shown. Horizontal dashed (green) lines represent zero values.

dissipation scale is resolved, and examined field lines at the grid scale for adequate microscopic smoothness. In Sect. 6 , we will further evidence how important is the numerical accuracy in studying the problem of reconnection in turbulence.

\subsection{The link between magnetic reconnection and turbulence}

Now we will take a closer look at the reconnection sites, trying to link them to the characteristic scales of MHD turbulence. Because of the complexity of the geometry we will focus only on the $X$-lines with higher reconnection rates, identified as described above. We need at this point to find a methodology to quantitatively characterize every reconnection region and extrapolate important information such as $\delta$ and $\ell$. Since we know the ratio of the eigenvalues obtained from the Hessian matrix analysis, using Eq. (6), the problem reduces to find just one of these lengths, say $\delta$. In Fig. 8a an example of the current density profile along the $s$ direction is shown. A consequence of the asymmetric nature of turbulent reconnection implies that in most of the cases 

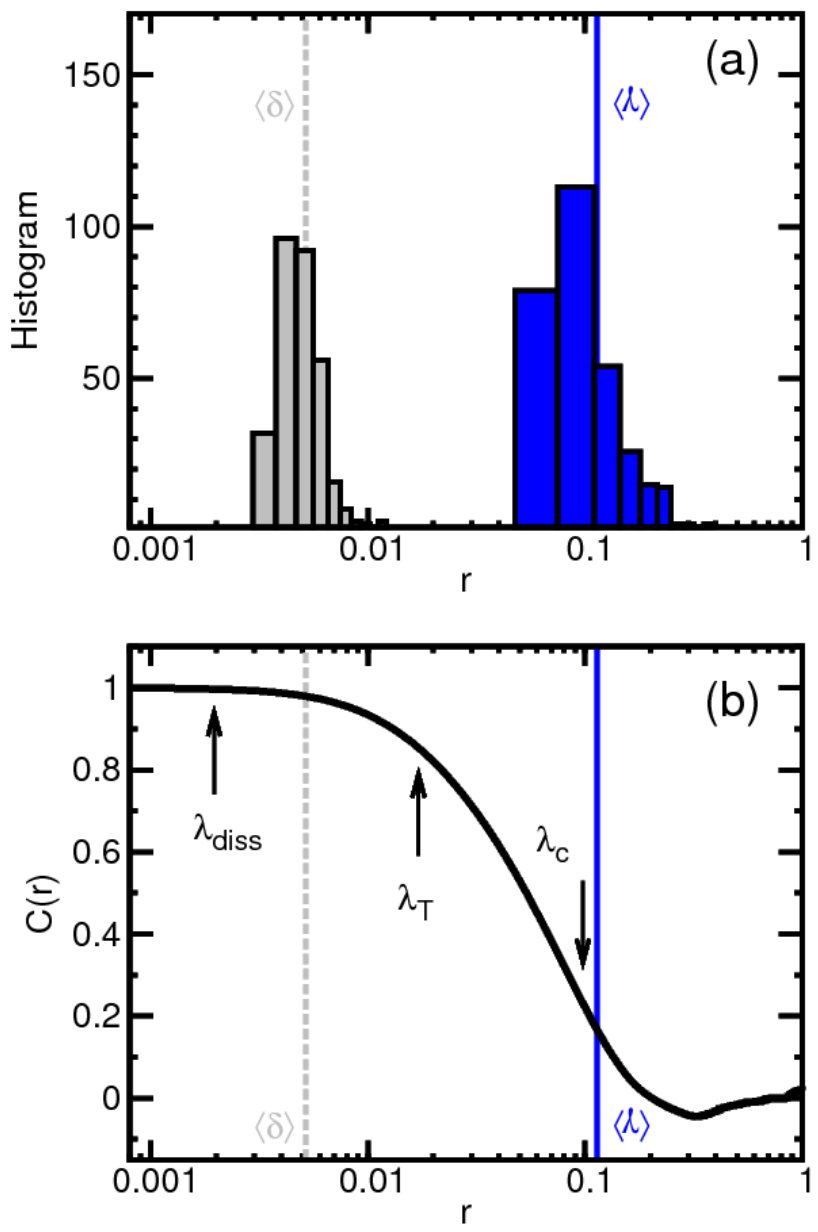

Fig. 9. (Color online) (a) histograms of thicknesses ( $\delta$, gray bars) and elongations $(\ell$, blue bars). Vertical lines are average values $\langle\delta\rangle$ (dashed gray) and $\langle\ell\rangle$ (full blue). (b) the magnetic field autocorrelation function (solid black line) is represented. The arrows (left to right) represent respectively: dissipation scale $\lambda_{\text {diss }}$, Taylor micro-scale $\lambda_{T}$ and correlation length $\lambda_{C}$. These characteristic scales are defined in Eq. (9) and text below.

the current density has a peak not centered precisely on the $X$-point (Cassak and Shay, 2007).

We call $b_{\mathrm{t}}(s)$ and $b_{\mathrm{n}}(s)$ the normal and the tangential component of the magnetic field, respectively. These components are obtained by projecting the in-plane magnetic field into the system of reference given by $\left\{\hat{\boldsymbol{e}}_{l}, \hat{\boldsymbol{e}}_{s}\right\}$, that is:

$b_{\mathrm{t}}=\hat{e}_{l} \cdot \boldsymbol{b}, \quad b_{\mathrm{n}}=\hat{e}_{s} \cdot \boldsymbol{b}$.

In Fig. 8b an example of the projected fields is reported. Note that a pile up of the magnetic field, in the upstream region of the reconnection event, is observed (Dorelli and Birn, 2003; Craig and Watson, 1999; Bodgan, 1984; Zweibel, 1995). Flux pile-up reconnection has been observed recently in an experiment on colliding flux ropes (Intrator et al., 2009). Using the eigensystem of the Hessian matrix $\left(\lambda_{i}\right.$
Table 2. Table of characteristic lengths (see text for details).

\begin{tabular}{rrrrrr}
\hline & $\begin{array}{r}\lambda_{c} \\
\left(\times 10^{-1}\right)\end{array}$ & $\begin{array}{r}\lambda_{T} \\
\left(\times 10^{-2}\right)\end{array}$ & $\begin{array}{r}\lambda_{\text {diss }} \\
\left(\times 10^{-3}\right)\end{array}$ & $\begin{array}{r}\langle\ell\rangle \\
\left(\times 10^{-1}\right)\end{array}$ & $\begin{array}{r}\langle\delta\rangle \\
\left(\times 10^{-2}\right)\end{array}$ \\
\hline Run 1 & 2.27 & 4.80 & 5.10 & 2.80 & 1.44 \\
Run 2 & 0.97 & 2.10 & 2.90 & 1.26 & 0.77 \\
Run 3 & 2.36 & 4.40 & 4.10 & 2.32 & 1.20 \\
Run 4 & 0.97 & 1.71 & 1.85 & 1.00 & 0.53 \\
Run 5 & 1.18 & 2.15 & 2.00 & 1.13 & 0.62 \\
Run 6 & 0.95 & 1.28 & 1.11 & 0.90 & 0.36 \\
\hline
\end{tabular}

and $\hat{\boldsymbol{e}}_{i}$ ), together with local fit-functions, the up-stream magnetic field can be estimated, locally, for each reconnection region. Note that, the process of reconnection in turbulence is often asymmetric (Cassak and Shay, 2007), so we define two upstream magnetic fields $b_{1}$ and $b_{2}$ (we suppressed subscript $t$ ).

The PDFs of $\delta$ and $\ell$ are reported in Fig. 9a, showing that they are well separated. We computed the mean values for these two lengths and we have found, for Run $4,\langle\delta\rangle \simeq 5 \times$ $10^{-3}$ and $\langle\ell\rangle \simeq 0.1$. These averages are reported, for all runs, in Table 2.

The present goal is to look for possible links between the reconnection geometry and the statistical properties of turbulence. In order to get more information about these associations we computed the auto-correlation function of the magnetic field. The correlation length is defined as

$\lambda_{C}=\int_{0}^{*} C(r) d r$,

where

$C(\mathbf{r})=\frac{\langle\boldsymbol{b}(\boldsymbol{x}+\mathbf{r}) \cdot \boldsymbol{b}(\boldsymbol{x})\rangle}{\left\langle b^{2}\right\rangle}$,

where the direction of displacement $r$ is arbitrary for isotropic turbulence in the plane, and the upper limit is unimportant if the distant eddies are uncorrelated. The correlation length $\lambda_{C}$ is a measure of the size of the energy containing islands. The auto-correlation function is illustrated in Fig. 9b. In the same figure $\langle\delta\rangle,\langle\ell\rangle$ are reported as vertical lines for comparison. The dissipation length, at which the turbulence is critically damped, is defined as $\lambda_{\text {diss }}=R_{\mu}^{-\frac{1}{2}}\left\langle\omega^{2}+j^{2}\right\rangle^{-\frac{1}{4}}$, while the Taylor micro-scale, a measure of mean-square gradients, is $\lambda_{T}=\sqrt{\frac{\left\langle\left.\boldsymbol{b}\right|^{2}\right\rangle}{\left\langle j^{2}\right\rangle}}$. The above lengths are represented in Fig. 9-(b).

It appears that the average elongation $\ell$ is strongly related to the correlation length where $C(r) \rightarrow 0$. As reported in Table 2, for all simulations, we found that the values of diffusion layer thickness $\delta$ is distributed in the range between the Taylor scale and the dissipation scale, while the length $\ell$, though broadly scattered, scales with $\lambda_{C}$ (cf. Fig. 9). The main features of this ensemble of reconnecting events, including the key length scales, are evidently controlled by the 


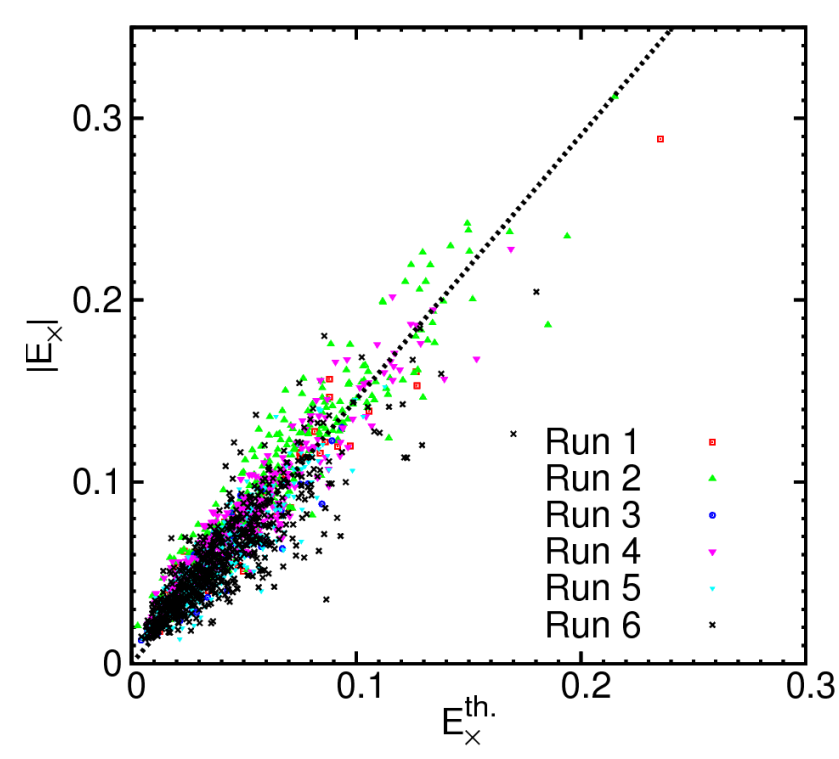

Fig. 10. (Color online) Computed reconnection rates vs. expectation from Eq. (11) (Cassak and Shay, 2007). The good agreement indicates that the system is reconnecting in a asymmetric SweetParker scenario.

statistical properties of turbulence, setting the range of values of length and thickness of the diffusion regions according to the correlation length and the dissipation scale. Note that a correlation between diffusion width and dissipation was discussed experimentally by Sundkvist et al. (2007).

\subsection{Generalized Sweet-Parker theory for reconnection in turbulence}

The turbulent reconnection activity identified above takes place in an environment in which the symmetric local conditions envisioned in standard laminar models are unlikely. It is therefore appropriate to employ the extension of the standard picture to asymmetric configurations. The SweetParker-type analysis for asymmetric anti-parallel reconnection has been studied in an earlier work by Cassak and Shay (2007). In particular, this analysis allows the reconnecting magnetic field strengths and plasma densities to be different on opposite sides of the dissipation region. Here we will summarize some of their main results. Asymmetric reconnection has also received recent attention in observations (Mozer and Pritchett, 2009; Mozer and Hull, 2010) and kinetic simulations (Pritchett and Mozer, 2009).

In the incompressible case and in our notation, the CassakShay (Cassak and Shay, 2007) asymmetric reconnection rate is given by

$E_{\times}^{t h .} \simeq \sqrt{\frac{b_{1}^{\frac{3}{2}} b_{2}^{\frac{3}{2}}}{R_{\mu} \ell}}$.
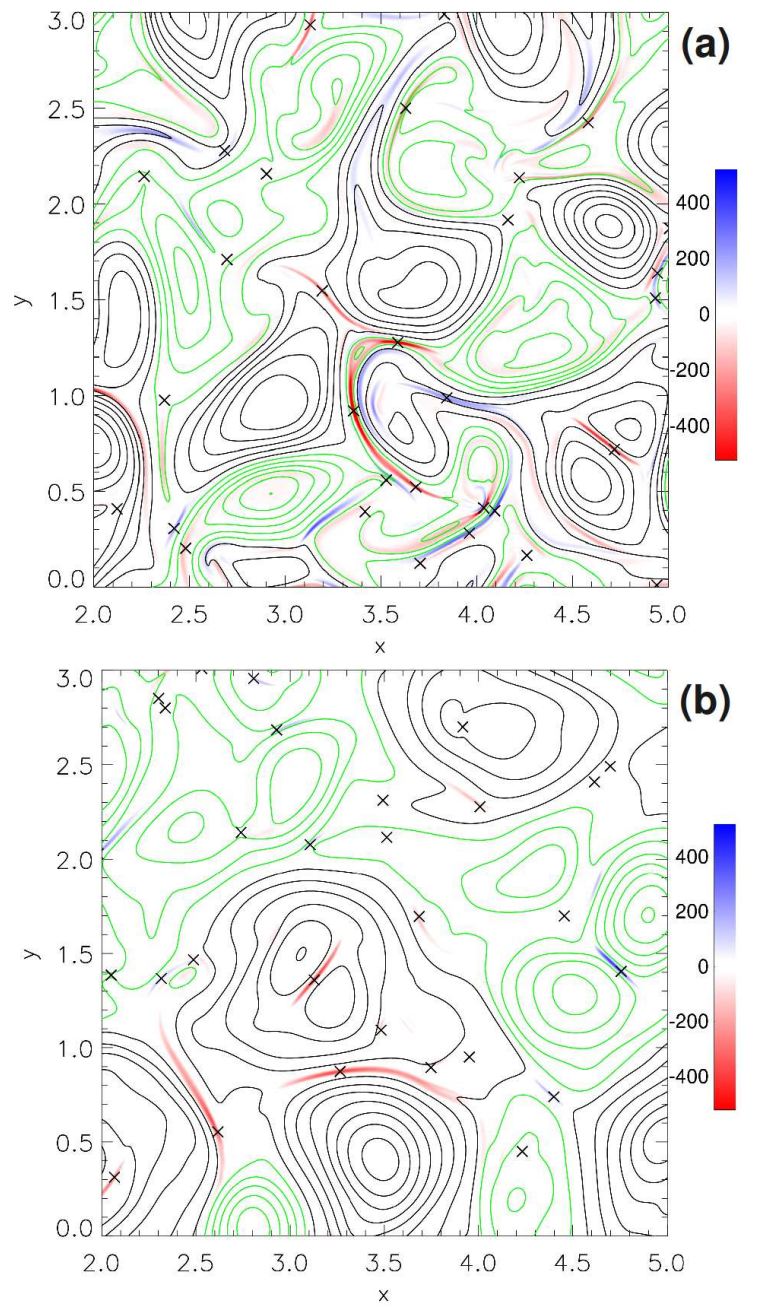

Fig. 11. (Color online) Shaded contour of the current density $j$ at different times of the simulations, namely $t=0.5$ (a) and $t=3.0$ (b). Iso-lines of the potential $a$ and the positions of the reconnection events (black crosses) are reported as well.

Here we examined whether the observed ensemble of turbulent reconnection events scales as asymmetric "SweetParker" in this sense, with resistivity causing the dissipation. To acquire a broader picture of the scaling, we evaluated Eq. (11) using several runs (listed in Fig. 10). Fig. 10 shows that in all the simulations the reconnection rates are consistent with the prediction given by Eq. (11). In this scenario turbulence plays a crucial role, determining locally the parameters that control the Sweet-Parker reconnection rate, namely, the lengths and local magnetic field strengths. Apparently, reconnection is an integral part of the turbulent cascade. 


\section{Time behavior of reconnection in turbulence}

In the previous sections we examined the statistics of magnetic reconnection in turbulence, at a given time $t^{*}$. The latter coincides with the peak of turbulent nonlinear activity, when the average current reaches its maximum (see Fig. 1). One may ask how the complex pattern of reconnection evolves in time, during the decaying evolution of 2-D MHD turbulence. In this brief section, we will answer the above question, simulating turbulence for longer times.

A moderate resolution of $4096^{2}$ mesh points will be chosen in order to reduce numerical costs. In analogy with simulations in Table 1, we impose random (Gaussian) fluctuations, for both velocity and magnetic fields, in the range $4 \leq k \leq 10$. The final time of the simulation is $t=3.0$, while the peak of the nonlinear activity is in this case reached at $t^{*} \sim 0.5$. At this time, as expected, all the features described in Sect. 3 are observed.

During the relaxation process, magnetic islands reconnect, merge, repel, and the system changes its magnetic topology. In Fig. 11 the current density is shown for two different times. The line-contours of the potential and the position of the $X$-points are superposed on the same figure. As it can be immediately noticed, the current sheets reduce both in number and intensity at $t=3.0$, where the turbulent pattern is characterized essentially by bigger islands. As an example, the number of reconnection sites at $t=0.5$ is 133 , reducing to 114 at the end of the simulation. This process will eventually continue in time until only few $X$-points survive to the turbulent evolution. Note that is difficult to explore this final stage of the relaxation, since it may occur after thousands of nonlinear times [see the long time behavior of the the MHD selective decay process in Matthaeus and Montgomery (1980) and the 2-D Navier Stokes analogy in Montgomery et al. (1992).]

In Fig. 12 we compare the reconnection rates at different instants of the simulation, namely at the beginning $(t=0)$, at the peak of nonlinear activity $(t=0.5)$, and at the end of the run $(t=3.0)$. Note that the reconnection rates have been properly normalized to $\delta b_{\mathrm{s}}(t)^{2}$ (see Sect. 3), at each time. In analogy with the time-behavior of the total current (see Fig. 1), the reconnection rates are higher when the turbulent activity is at the peak. This further provides evidence that fully developed turbulence and fast reconnection events may strongly be related in plasma dynamics.

Previously, we found that the strongest reconnection rates depend on the local geometry of the diffusion region. In particular, as shown in Fig. 7, the reconnection rate scales with the aspect ratio $\ell / \delta$ (see Eq. (7 and discussion). In Fig. 13a we show the same scatter plot at different times. At the beginning of the simulation, since initial conditions are Gaussian, there is no clear scaling. This is related to the absence of coherency in the magnetic field (see Fig. 7 and Wan et al., 2009). After the peak of the activity is reached, for $t>0.4$, the expectation given by Eq. (7) is recovered. At later times,

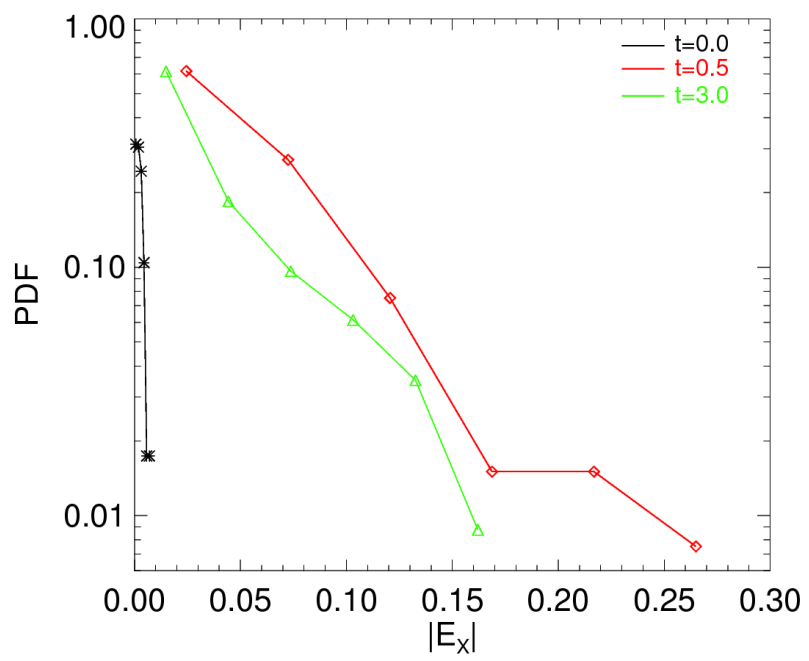

Fig. 12. (Color online) Time evolution of the PDF of $\left|E_{\times}\right|$, namely at $t=0.0$ (black stars), 0.5 (red open diamonds), and $t=3.0$ (green open triangles).

the fastest reconnection events, with geometries characterized by large aspect ratios, vanish.

In Fig. 13b, the reconnection rates are compared, at different times, with the asymmetric Sweet-Parker prediction given by Eq. (11). It is evident that the model nicely describes the process of reconnection in 2-D MHD turbulence: during the time relaxation of turbulence, the reconnection events still obey the theory proposed by Cassak and Shay (2007).

\section{Preliminary results with the Hall effect}

Besides turbulence, an ingredient that may accelerate the process of reconnection is the Hall effect. The latter becomes crucial when the ion skin depth, defined as $d_{i}=c / \omega_{p i}$ (being $\omega_{p i}$ the ion plasma frequency and $c$ the speed of light), is a non-negligible fraction of the system size $L_{0}$, namely when $d_{i} / L_{0} \neq 0$ (Roberts and Taylor, 1962). Generally, the Hall effect is thought to be crucial in plasmas, since it cause small scale turbulent activity, producing a departure from power spectra predictions of the MHD theory (Servidio et al., 2007; Galtier and Buchlin, 2007; Dmitruk and Matthaeus, 2006; Mininni et al., 2007; Matthaeus et al., 2008; Alexandrova et al., 2007), and, when $d_{i} / L_{0}$ is large enough, changes in the decay rate of the turbulence at moderate Reynolds number (Matthaeus et al., 2003). In the past years, the role of the ion skin depth on reconnection has been matter of several numerical investigations (Ma and Bhattacharjee, 2001; Smith et al., 2004; Lu et al., 2010). In particular, it has been proposed that the Hall effect in reconnection cause a catastrophic release of magnetic energy, leading to fast magnetic reconnection onset (Cassak et al., 2005, 2007), with reconnection rates faster 


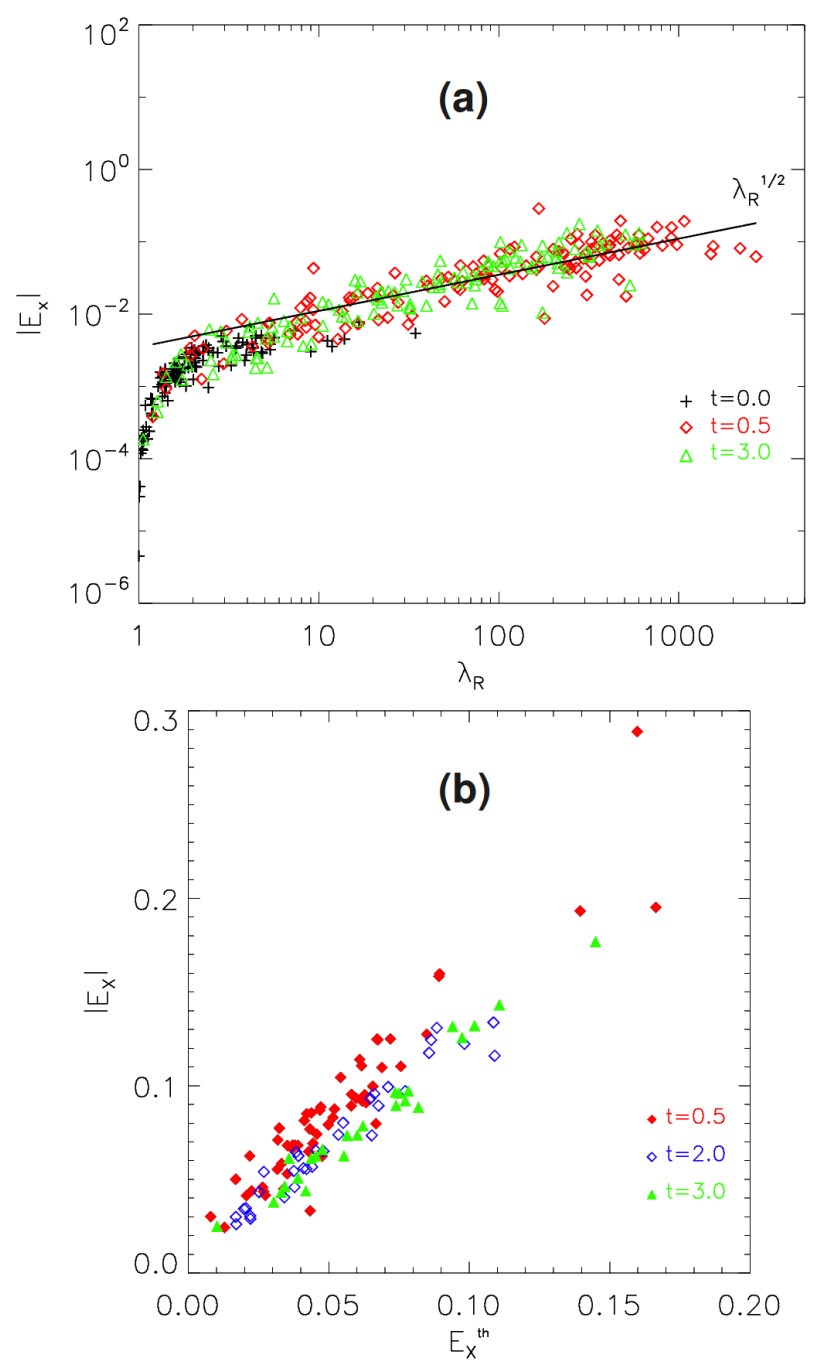

Fig. 13. (a) Relation between the reconnection rates $\left|E_{\times}\right|$and the geometry of the reconnection region $\lambda_{R}$, for different times of the simulation. After the peak of the nonlinear activity, the scaling $\lambda_{R}^{1 / 2}$ (green solid line) appears. (b) Asymmetric Sweet-Parker prediction at different times of the simulation. This theory accurately describes the dynamics of reconnection in 2-D MHD turbulence.

Table 3. Table of runs. The second column is the resolution of the simulation, third column the Reynolds numbers, fourth column reports the dissipative scale of the system and the last column shows the Hall parameter.

\begin{tabular}{llllll}
\hline Run & Eqs. & resolution & $R_{\mu}\left(R_{\nu}\right)$ & $\lambda_{\text {diss }}$ & $\epsilon_{H}\left(k_{H}^{-1}\right)$ \\
\hline I & MHD & $4096^{2}$ & 1700 & $1 / 200$ & 0 \\
II & Hall MHD & $4096^{2}$ & 1700 & $1 / 200$ & $1 / 100$ \\
\hline
\end{tabular}

than the Sweet-Parker expectation. In this short section we will inspect statistically the role of the Hall effect on the process of reconnection in turbulence.

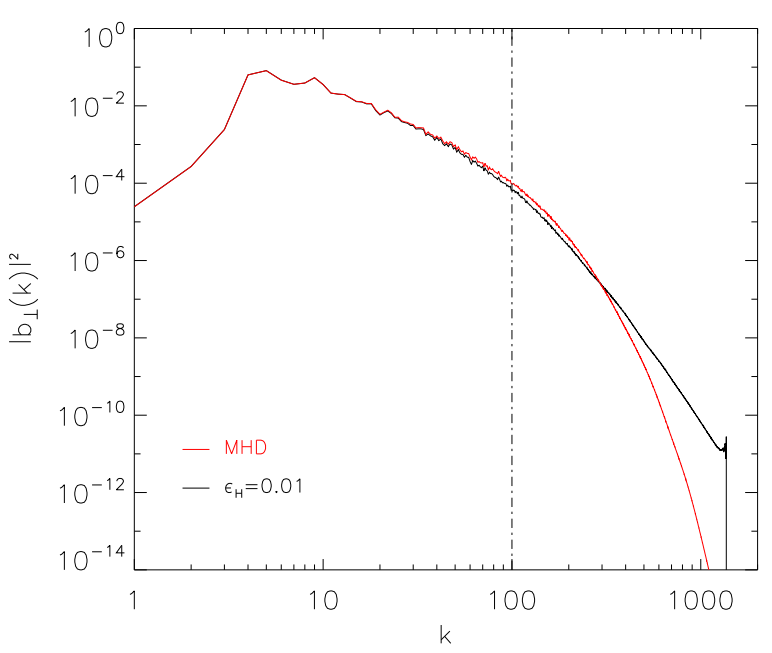

Fig. 14. Power spectra of the "perpendicular" magnetic energy for MHD (red) and Hall MHD (black). The vertical dashed line represent the Hall wavenumber $k_{H}=\epsilon_{H}^{-1}$.

Analogously to MHD, the equations of incompressible Hall MHD can be written in dimensionless form. In 2.5$\mathrm{D}$ (2 dimensions in the physical space for three-dimensional components) the equations read:

$\frac{\partial \boldsymbol{v}}{\partial t}=-(\boldsymbol{v} \cdot \nabla) \boldsymbol{v}-\nabla P+\boldsymbol{j} \times \boldsymbol{b}+R_{v}^{-1} \nabla^{2} \boldsymbol{v}$,

$\frac{\partial \boldsymbol{b}}{\partial t}=\boldsymbol{\nabla} \times\left[\left(\boldsymbol{v}-\epsilon_{H} \boldsymbol{j}\right) \times \boldsymbol{b}\right]+R_{\mu}^{-1} \nabla^{2} \boldsymbol{b}$.

The fields can be decomposed in perpendicular (in-plane) and parallel (out-of-plane, along $z$ ) components, namely $\boldsymbol{b}=\left(\boldsymbol{b}_{\perp}, b_{z}\right)$ and $\boldsymbol{v}=\left(\boldsymbol{v}_{\perp}, v_{z}\right)$. For the magnetic field $\boldsymbol{b}_{\perp}=$ $\nabla a \times \hat{z}$, where $a$ is the magnetic potential. The coefficient $\epsilon_{H}=d_{i} / L_{0}$ is the Hall parameter and is proportional to the amount of dispersive effects present in the system. Note that, for $\epsilon_{H} \rightarrow 0$, Eqs. (12)-(13) reduce to MHD (see Eqs. 1-2). The above equations are solved with the same algorithm used for the MHD case. We performed 2 simulations, MHD and Hall MHD, summarized in Table 3.

In order to quantify the differences between MHD and Hall MHD turbulence, we compare the power spectra for $\boldsymbol{b}_{\perp}$ (in-plane components), reported in Fig. 14. The main difference between the two runs can be noticed at small scales, namely at $k>k_{h}$. This difference consists of a higher small scale activity in the Hall case, and is generally attributed to the presence of dispersive effects (Servidio et al., 2007).

As we said before, the current density is an important quantity since it captures many of the small scale features in both turbulence and in reconnection. We show in Fig. 15 the PDF of $j_{z}$ (out of plane component), for the runs in Table 3. The core of the distributions is very similar for both 


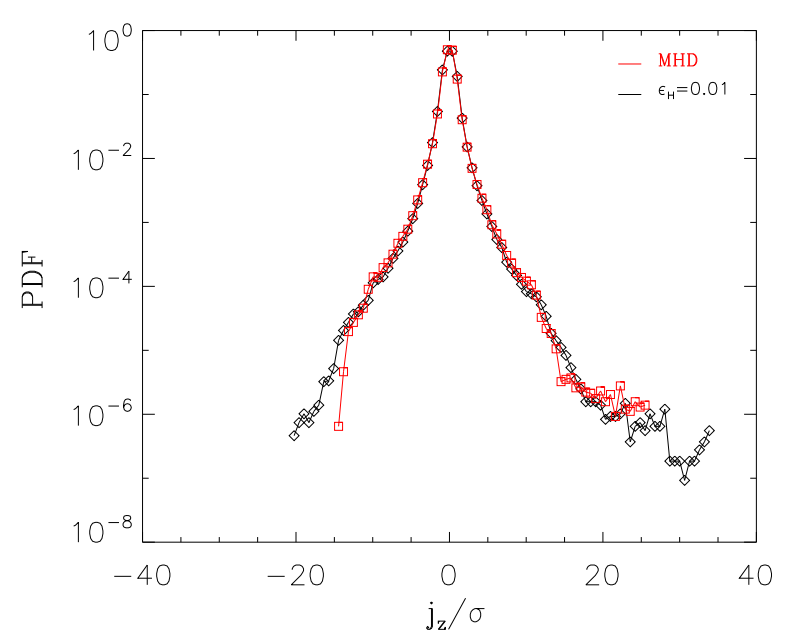

Fig. 15. PDF of $j_{z}$, normalized to its rms value, for MHD (red) and Hall MHD (black). The longer tails present in the Hall case are the signature of a more intense small-scale activity.

simulations, but in the Hall MHD cases the tails are more pronounced. This suggests that dispersive effects cause an enhancement of the small scale activity. We will further investigate this interesting statistical property of Hall MHD turbulence in upcoming publications.

The PDF of $E_{\times}$, for both MHD and Hall MHD, are reported in Fig. 16. As expected, both distributions manifest strong departures from the averages. Since for these runs we did not have enough events ( $X$-points), the PDFs have been constructed using constant weight $m$ per-bin (variable amplitude PDF), with $m=6$ (Osman et al., 2011; Bruno and Carbone, 2005). In the Hall case, a higher tail appearing in the PDF indicates that the Hall term slightly accelerates reconnection. As an example, for MHD we obtained the mean value $\left\langle\left|E_{\times}\right|\right\rangle \simeq 0.05$, while for Hall $\mathrm{MHD}\left\langle\left|E_{\times}\right|\right\rangle \simeq 0.06$. This analysis confirms that when the Hall physics is present, distributions of reconnection rates become broader than the MHD case, leading to slightly faster rates on average, but a net increase in the frequency of occurrence of the fastest rates. Note that in the Hall MHD simulation the maximum reconnection rate goes up to $\left|E_{\times}\right| \sim 0.38$. We believe, on the other hand, that further investigation is needed. More simulations, varying for example the strength of the Hall effect, and more events will clarify how the process of reconnection in turbulence is affected by dynamical activity at the ion skin depth. A more detailed study will be presented in a future work.

\section{Warning about the accuracy of simulations}

In this section we will briefly review the widely recognized issue of adequate spatial resolution in numerical simulations

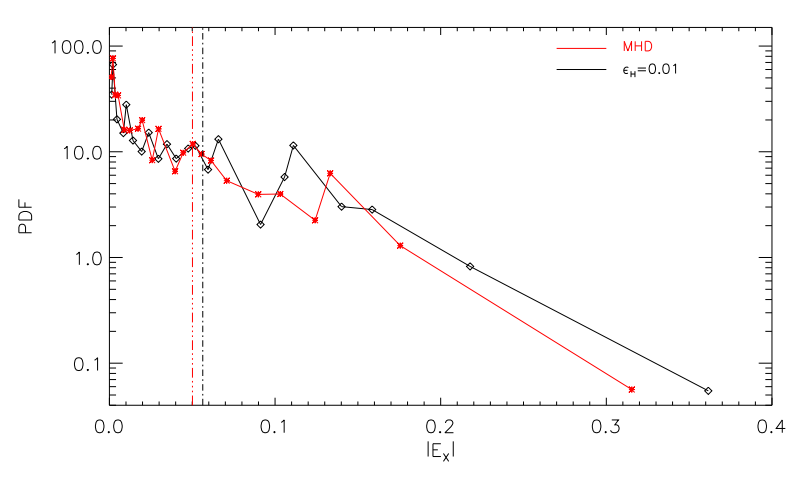

Fig. 16. (Color online) Probability distribution function of reconnection rates in MHD (red) and Hall MHD (black) turbulence. In the Hall case, the population of strong reconnection events is higher. Vertical lines represent averages.

of turbulence, in the context of 2-D MHD (Donzis et al., 2008; Wan et al., 2010). Generally, it is widely thought that a good criterion for the quality of numerics is given by the spectra: a nice power spectrum is synonym of a good simulation. Here we argue that this is not sufficient, since a slightly inadequate resolution fails for precise determination of higher-order statistical quantities, and therefore fails to accurately describe reconnection. In particular, in Wan et al. (2010) it was suggested that oversampling the Kolmogorov dissipation scale $\eta$ by a factor of 3 allows accurate computation of the kurtosis, the scale-dependent kurtosis, and the reconnection rates (see Eq. (14 for a definition of $\eta$ ). These tests may provide useful guidance for resolution requirements in many plasma computations involving turbulence and reconnection.

We begin with the assumption that stable computation of the fourth-order moments is desirable. A more sensitive test involving scale-dependent kurtosis is also found to be indicative. To understand the utility of these ideas we examine the problem in which the quantity of interest is the distribution of magnetic reconnection rates in MHD turbulence. Since many reconnection sites occur, we are particularly interested in the conditions on spatial resolution that must be attained to accurately compute the tail of this distribution, because this tail measures the likelihood of the highest rates of reconnection. It turns out that the same conditions for accurately computing the fourth-order moments also give rise to accurate computation of reconnection rates.

Here we provide a numerical example, in which a series of numerical runs is carried out, beginning with identical initial data and dissipation coefficients, but with varying spatial resolution. As described in Sect. 2, we make use of spectral method simulations, with $N$ Fourier modes in each Cartesian direction (Ghosh et al., 1993; Canuto et al., 1988). All runs are performed with $v=\mu=1 / 2000$ and are unforced. The runs are dealiased, using the Orszag-Patterson approach with $k_{\max }=\sqrt{2} N / 3$ and phase-shift dealiasing (Patterson 
Table 4. Parameters for the simulations. The initially excited Fourier modes have $5<|\boldsymbol{k}|<30$, and $k_{\text {diss }}$ means the maximum value of $k_{\text {diss }}(t)$.

\begin{tabular}{lrrrrl}
\hline Run & Grid & $R e$ & $k_{\max }$ & $k_{\text {diss }}$ & $\frac{k_{\max }}{k_{\text {diss }}}$ \\
\hline 1 & $256^{2}$ & 2000 & 120 & 242 & 0.50 \\
2 & $512^{2}$ & 2000 & 241 & 250 & 0.96 \\
3 & $1024^{2}$ & 2000 & 482 & 251 & 1.9 \\
4 & $1536^{2}$ & 2000 & 724 & 251 & 2.9 \\
5 & $2048^{2}$ & 2000 & 965 & 251 & 3.9 \\
6 & $4096^{2}$ & 2000 & 1930 & 251 & 7.7 \\
\hline
\end{tabular}

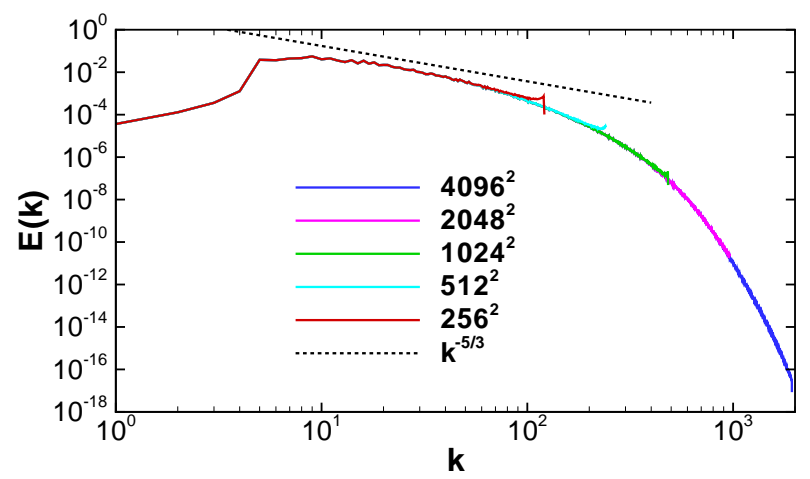

Fig. 17. (Color) Energy spectra at the time of maximum kurtosis $\chi_{j}$, for all runs. A $k^{-5 / 3}$ powerlaw (dotted) is shown for comparison. The $1536^{2}$ run is not plotted to avoid clutter.

and Orszag, 1971). We remind that the dissipation wavenumber (reciprocal of the Kolmogorov scale $\eta$ ) is defined as

$\operatorname{kdiss}(t) \equiv \eta^{-1}=\left(\frac{\epsilon}{\nu^{3}}\right)^{1 / 4} \equiv \frac{\left\langle\omega^{2}+j^{2}\right\rangle^{1 / 4}}{\sqrt{v}}$,

where $\langle\cdots\rangle$ denotes spatial averaging and $\epsilon$ is the average rate of energy dissipation. The ratio $k_{\max } / k_{\mathrm{diss}}(t) \equiv k_{\max } \eta$ can be interpreted as a measure of adequate resolution of the dissipation scale, with larger values indicating better resolution. Below, we make regular use of $k_{\max } / k_{\text {diss }}$ as an organizing parameter for the simulations. All the runs are summarized in Table 4.

Figure 17 shows the energy spectra for the runs listed in Table 4. For each run, the spectrum is computed at the time of maximum current kurtosis $\chi_{j}$. The results show that all the spectra agree well. Indeed, although the spectra cut off at different wavenumbers (because of the different run resolutions), the curves nearly overlay each other over the full range of overlapping $k$, with only small discrepancies in the lower resolution runs near their maximum retained wavenumber. The importance of this comparison is that an examination of the "quality" of an energy spectrum is a frequently encountered approach to heuristically evaluate the

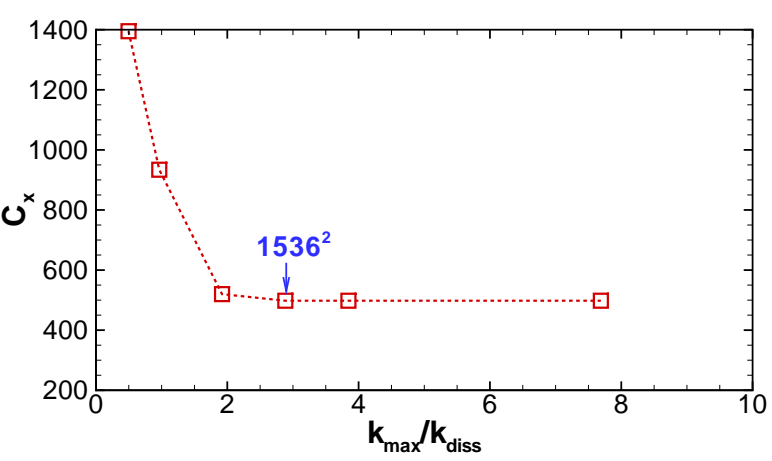

Fig. 18. (Color online) Number of reconnection sites $C_{\times}$as a function of $k_{\max } / k_{\text {diss }}$, for the simulations in Table 4 . For this choice of the viscous terms, $v=\mu=1 / 2000$, the saturation occurs at resolution of $\sim 1536^{2}$.

quality of spatial resolution. However, we now show that spectra can be reasonably accurate even when other quantities are not.

Figure 18 displays the number of reconnection sites $C_{\times}$ as a function of $k_{\max } / k_{\text {diss. }}$. It is evident that under-resolved runs include many additional $X$-points that are not found in the well-resolved cases. Evidently the phase errors caused by under-resolution imply a lack of intermittency, and also a spurious increase in the number of $X$-points present, due to Gaussianization of the fluctuations (for more details see Wan et al., 2009). However, a clear saturation in $C_{\times}$occurs once $k_{\max } / k_{\mathrm{diss}}$ becomes large enough. In Fig. 19 we show contour plots of the magnetic potential $a$ together with the positions of reconnection sites, for both well-resolved (Run 6) and under-resolved (Run 1) simulations. In the underresolved case a higher number of $X$-points is present. Note that this excess of $X$-points, due to small-scale Gaussianization process, can be easily confused with real (physical) phenomena such as secondary tearing instabilities (Daughton et al., 2009; Wang et al., 2010), current-sheet disruptions or chain of plasmoids formation.

Another important feature is the dependency of the reconnection rates on the accuracy of the simulations. In Fig. 20 the PDFs of $\left|E_{\times}\right|$are shown. Comparing results from different runs, one observes that the PDFs for the under-resolved runs manifest much shorter tails. As the resolution increases, the tails become broader until saturation occurs, at $\sim 2048^{2}$. These results further confirm our statements: if the resolution is insufficient, then the reconnection is not properly treated in the simulations.

\section{Applications to the turbulent solar wind}

The statistical properties of reconnection have been investigated in the previous sections, leading to the conclusion that strong reconnection events can locally occur in 2-D MHD turbulence. In this section we will review some of the main 

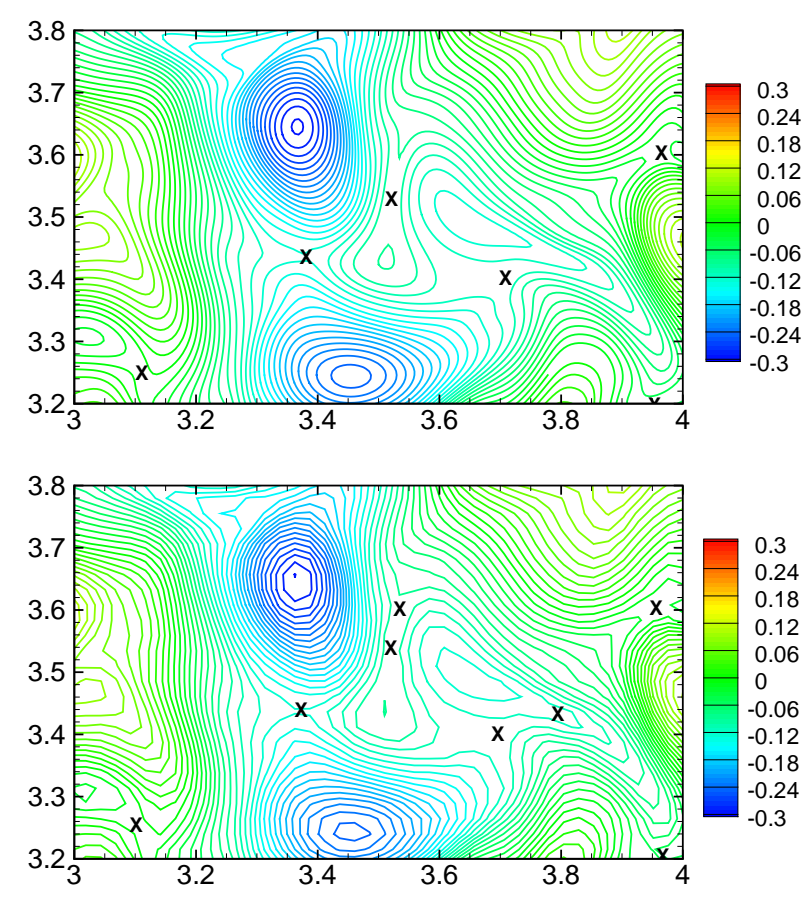

Fig. 19. (Color online) Contour plots of the magnetic potential $a$ with the positions of reconnection sites ( $X$-points) in black $\times$ from a well-resolved run (Run 6, top) and a under-resolved run (Run 1, bottom).

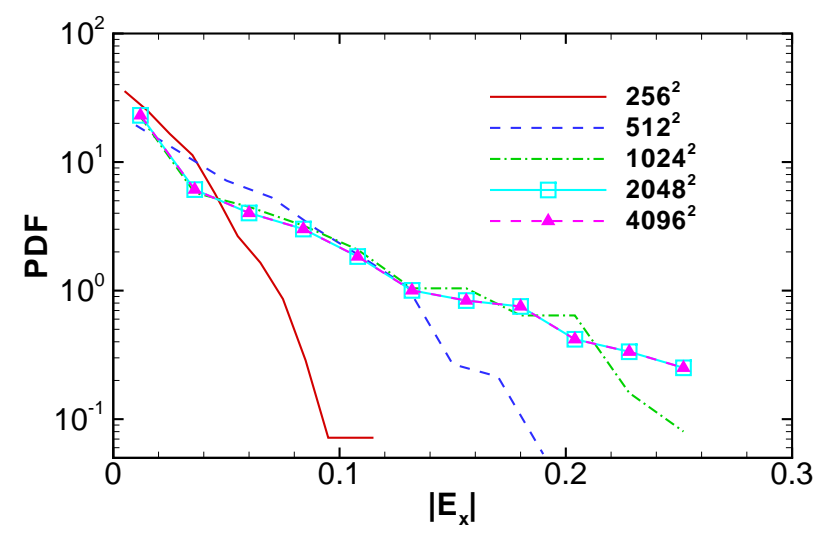

Fig. 20. (Color online) PDFs of the reconnection rate $\left|E_{\times}\right|$for all the runs. In the case of unresolved simulations, the reconnection rates dramatically reduce.

results about the link between solar wind discontinuities and local magnetic reconnection processes. A well-known feature of solar wind observations is, in fact, the appearance of sudden changes in the magnetic field vector, defined as directional discontinuities (DDs), which are detected throughout the heliosphere (Burlaga, 1968; Tsurutani and Smith, 1979; Ness and Burlaga, 2001; Neugebauer, 2006; Bruno et al., 2001; Sorriso-Valvo et al., 1999). These changes are often

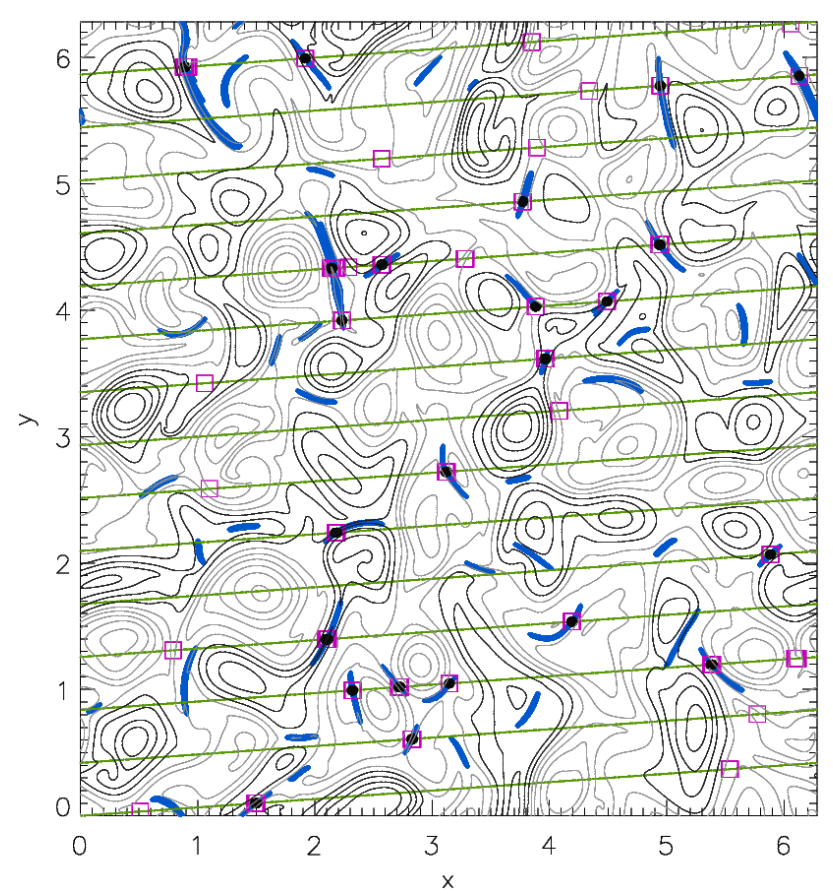

Fig. 21. Contour lines of the magnetic field (or line contour of $a$ ) together with the diffusion regions (blue shaded map), and with the one-dimensional path $s$ (green solid line). On the same plot, the discontinuities identified by PVI technique with a threshold $\theta=5$ in Eq. (16) (open magenta squares) are represented. Bullets (black) are discontinuities which correspond to reconnection sites.

seen at time-scales of 3 to 5 minutes, although similar discontinuities are seen at smaller time scales (Vasquez et al., 2007). In addition to identification based on characterization of discontinuities, coherent structures have also been identified using other approaches, such as wavelets (Veltri and Mangeney, 1999; Bruno et al., 2001), phase coherency analysis (Hada et al., 2003; Koga and Hada, 2003; Koga et al., 2007) and "surrogate data" analysis (Sahraoui and Goldstein, 2010; Sahraoui, 2008).

One interpretation of magnetic discontinuities is that they are the walls between filamentary structures of a discontinuous solar wind plasma (Burlaga, 1969; Borovsky, 2006; Borovsky and Denton, 2010; Bruno et al., 2001), while another is that some strong discontinuities are fossils from the birth of the solar wind (Burlaga, 1968; Borovsky, 2008). An alternative possibility is that the observed discontinuities are the current sheets that form as a consequence of the MHD turbulent cascade (Matthaeus and Montgomery, 1980; Veltri, 1999). Recent studies on magnetic discontinuities show that their statistical properties are very similar to distributions obtained from simulations of MHD turbulence (Greco et al., 2008, 2009). This line of reasoning argues that thin current sheets are characteristic coherent structures expected in active intermittent MHD turbulence (Mininni and Pouquet, 

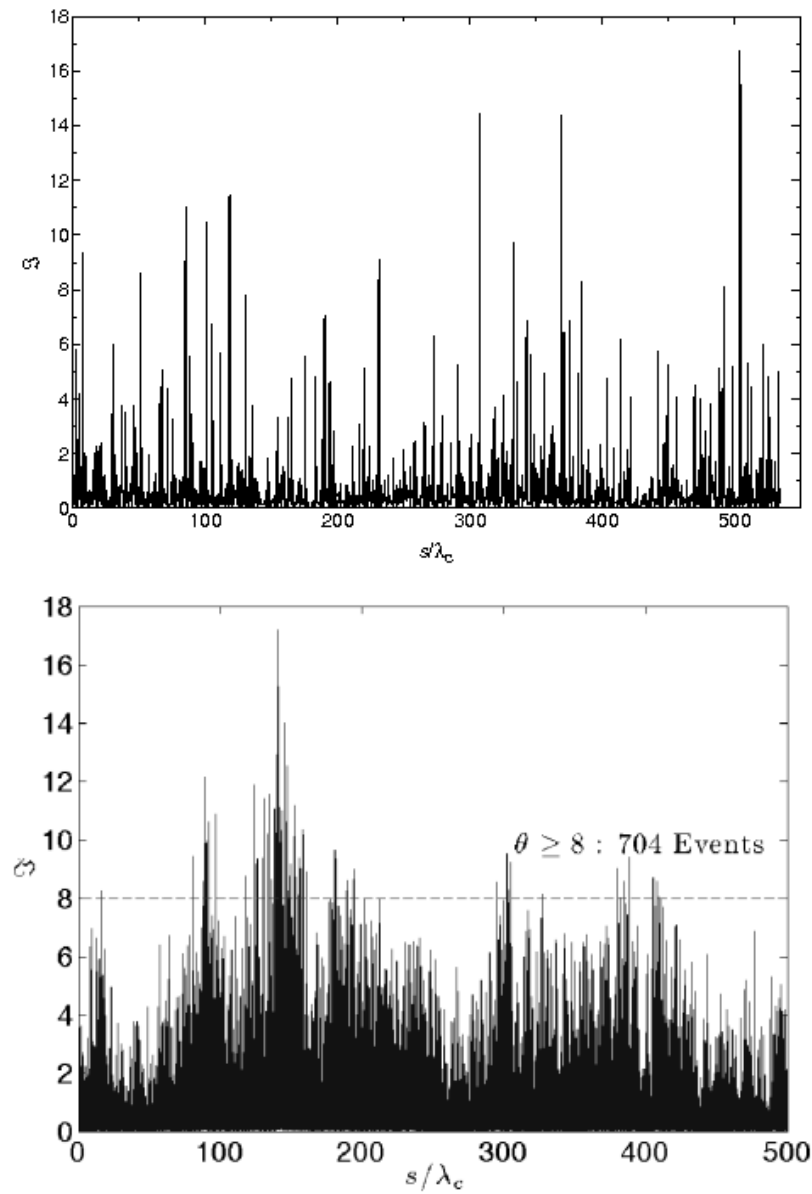

Fig. 22. Top: spatial signal $\Im(\Delta s, \ell, s)$ (PVI) obtained from the simulation by sampling along the trajectory $s$ in the simulation box, with $\Delta s \simeq 0.67 \lambda_{d}$ and $\ell \simeq 535 \lambda_{C}$. Bottom: same quantity obtained from solar wind data, with $\Delta s=20 \mathrm{~s}$ and $\ell \simeq 500 \lambda_{C}$.

2009), and which are therefore integral to the dynamical couplings across scales. Therefore, solar wind discontinuities are one of the best applications of our theory of reconnectionin-turbulence.

In this perspective, one is led naturally to suspect that at least some of the current sheets that are a common feature of the solar wind at $1 \mathrm{AU}$ may be participating in small-scale magnetic reconnection (Sundkvist et al., 2007; Gosling and Szabo, 2008; Phan et al., 2010), as well as inhomogeneous interplanetary plasma dissipation and heating (Leamon et al., 2000; Osman et al., 2011). To further establish the relationship between current sheets and small scale reconnection in turbulence, some quantitative connection is needed. We have in mind the particular question: if one identifies a current sheet in turbulence, how likely is it to be also an active reconnection site? Here we show, using MHD simulation data, that methods for identifying intermittent current sheet-like structures, when quantified properly, can identify sets of structures that are likely to be active reconnection regions.
For the present statistical analysis we will consider Run 1 described in Table 1. Anticipating possible applications to spacecraft data, we focus on properties of discontinuities that are recorded by magnetic field measurements at a single spacecraft in interplanetary space. We adopt a spacecraftlike sampling through the simulation domain (see Fig. 21 and Greco et al., 2008), and we call $s$ this trajectory. In particular, we can define a set whose elements consist of the segments of a trajectory that passes through any reconnection zone, identified by the cellular automaton method (Servidio et al., 2010a,b). In this way we can build a set of strong reconnection site encounters RS associated with a trajectory. Figure 21 shows an example of reconnection sites together with the one-dimensional path $s$.

Interpolating the magnetic field data along the onedimensional path $s$ Greco et al. (2008), we can identify discontinuities (TDs) with the following procedure:

1. First, to describe rapid changes in the magnetic field, we look at the increments

$$
\Delta \boldsymbol{b}(s, \Delta s)=\boldsymbol{b}(s+\Delta s)-\boldsymbol{b}(s),
$$

where $\Delta s$ the spatial separation or lag. For this simulation we choose a small scale lag, $\Delta s \simeq 0.67 \lambda_{\text {diss }}$, which is comparable to the turbulence dissipation scales (see previous sections).

2. Second, employing only the sequence of magnetic increments, we compute the normalized magnitude

$$
\Im(\Delta s, \ell, s)=\frac{|\Delta \mathbf{b}(s, \Delta s)|}{\sqrt{\left\langle|\Delta \boldsymbol{b}(s, \Delta s)|^{2}\right\rangle_{\ell}}},
$$

where $\langle\bullet\rangle_{\ell}=(1 / \ell) \int_{\ell} \bullet d s$ denotes a spatial average over an interval of length $\ell$, and $\Delta s$ is the spatial lag in Eq. (15). The square of the above quantity has been called the Partial Variance of Increments (PVI) (Greco et al., 2008) and the method abbreviated as the PVI method. For the numerical analysis performed here $\ell \simeq 535 \lambda_{C}$, where $\lambda_{C}=0.18$ is the turbulence correlation length - a natural scale for computing averages.

The PVI time series, evaluated using Eqs. (15)-(16) is reported in Fig. 22. The illustration spans more than 500 correlation lengths. This spatial signal has been compared to a time signal measured by a ACE solar wind spacecraft, near $1 \mathrm{AU}$, over a period of about 20 days (lower panel of the figure). In order to facilitate the comparison, we converted the time signal to a spatial signal, using the average velocity of the flow, and then normalized to a solar wind magnetic correlation length of $1.2 \times 10^{6} \mathrm{~km}$.

The PVI (actually "square-root PVI", hereafter just PVI) increment time series is bursty, suggesting the presence of sharp gradients and localized coherent structures in the magnetic field, that represent the spatial intermittency of turbulence. These events may correspond to what are qualitatively 
Table 5. First column: label of the method $\Im_{\theta}$. Second column: threshold $\theta$ imposed on PVI, cf. Eq. (17). Third column: \#ITD, number of discontinuities identified by the method. Fourth column: \#IRS, number of reconnection sites found by the method. Fifth column: \#IRS/\#RS, the relative efficiency of the method, identified reconnection sites as percent of all the reconnection sites present along the path. Last column: \#IRS/\#ITD, the relative goodness of the method, percent of identified reconnection events in set of identified discontinuities.

\begin{tabular}{cccccc}
\hline Method & $\theta$ & \# ITD & \# IRS & efficiency $(\%)$ & goodness $(\%)$ \\
\hline$\Im_{1}$ & 1 & 378 & 37 & 100 & 9.8 \\
$\Im_{5}$ & 5 & 40 & 23 & 62.2 & 57.5 \\
$\Im_{8}$ & 8 & 13 & 13 & 35.1 & 100 \\
\hline
\end{tabular}

called "tangential discontinuities" and, possibly, to reconnection events.

Imposing a threshold $\theta$ on Eq. (16), a collection of stronger discontinuities along the path $s$ can be identified. That is, we select portions of the trajectory in which the condition

$\Im(\Delta s, \ell, s)>\theta$

is satisfied, and we will employ this condition to identify candidate reconnection sites. In Fig. 21, an example of the location of discontinuities along $s$, selected by the PVI method with a particular threshold $\theta$, is shown. One can immediately see in Fig. 21 that there is an association, but not an identity, between the set of "events" identified using Eq. (17), and the encounters of the trajectory with reconnection regions. We will now study this association quantitatively using different values of threshold $\theta$. To understand the physical meaning of the threshold $\theta$, we recall Greco et al. $(2008,2009)$ that the probability distribution of the PVI statistic derived from a nonGaussian turbulent signal is empirically found to strongly deviate from the pdf of PVI computed from a Gaussian signal, for values of PVI greater than about 3. As PVI increases to values of 4 or more, the recorded "events" are extremely likely to be associated with coherent structures and therefore inconsistent with a signal having random phases. Thus, as $\theta$ is increased, stronger and more rare events are identified, associated with highly nonGaussian coherent structures.

We now adopt a procedure to count how many of the identified TDs (from Eq. 17) are also reconnection sites (i.e., elements of the set RS), as follows: every discontinuity is characterized by a starting and an ending point along the synthetic trajectory $s$. A set of discontinuities is identified, and a certain number of these discontinuities intersect reconnection regions. To automate the determination of the reconnection regions, we make use of a map (Servidio et al., 2010b) that is generated using the cellular automaton procedure. The latter, in summary, is a 2-D matrix that has 0 values in all cells outside of the diffusion regions, or values of 1 inside the diffusion regions. For this simulation, and for the selected trajectory (see Fig. 21), there are 37 reconnection sites along the

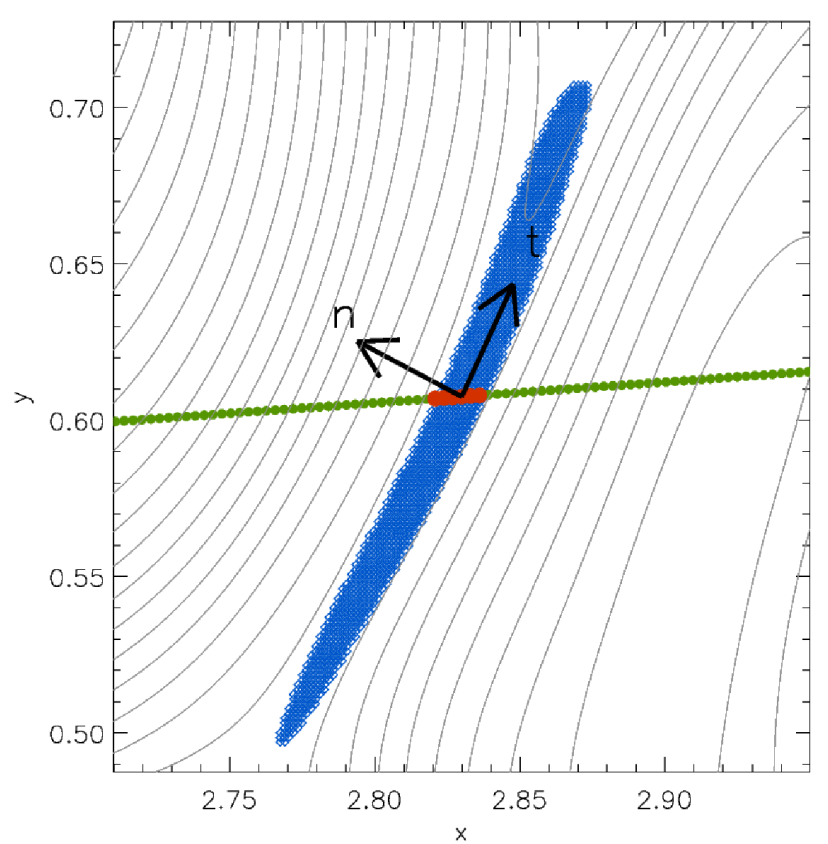

Fig. 23. Zoom into the tangential discontinuity: two-dimensional crossing of the reconnection site (blue region, same as Fig. 21), together with the trajectory (green bullets), the TD captured by the PVI technique (red dots) and the minimum variance reference (MVA) frame ( $\hat{\mathbf{n}}, \hat{\mathbf{t}})$ (black arrows).

path $s$. When at least one point of the identified candidate discontinuity overlaps with one point of the identified reconnection region, the event is counted as a "success". Otherwise the TD is not identified as an RS, and is a "failure". In the latter case the method is detecting a non-reconnecting, high-stress, magnetic field structure. However, such points are not associated with a region of strong reconnection, and therefore are not of interest in this analysis.

As an example, using $\theta=5$ in Eq. (17), 40 discontinuities have been identified and 23 overlap a reconnection site and correspond to successful identification of a reconnection region. The goodness (quality) of this method can be defined as the number of the successes over the total number of identified discontinuities. For this example, the goodness is $\simeq 57.5 \%$. An example of discontinuities, together with the reconnecting regions, is shown in Fig. 21.

Following the above procedure summarized by Eq. (17), we impose different threshold $\theta$ for the PVI signal. Each threshold characterizes a different set of discontinuities or "events", and we can label each algorithm as $\Im_{\theta}$. The parameters of different PVI-based algorithms are listed in Table 5, all of which use $\Im\left(\Delta s=0.76 \lambda_{d}, \ell=535 \lambda_{C}\right)$. It can be seen that for higher values of $\theta$ an increasing fraction of the identified TDs corresponds to a reconnection site. That is, the goodness increases as the threshold $\theta$ is increased (Servidio et al., 2011). 

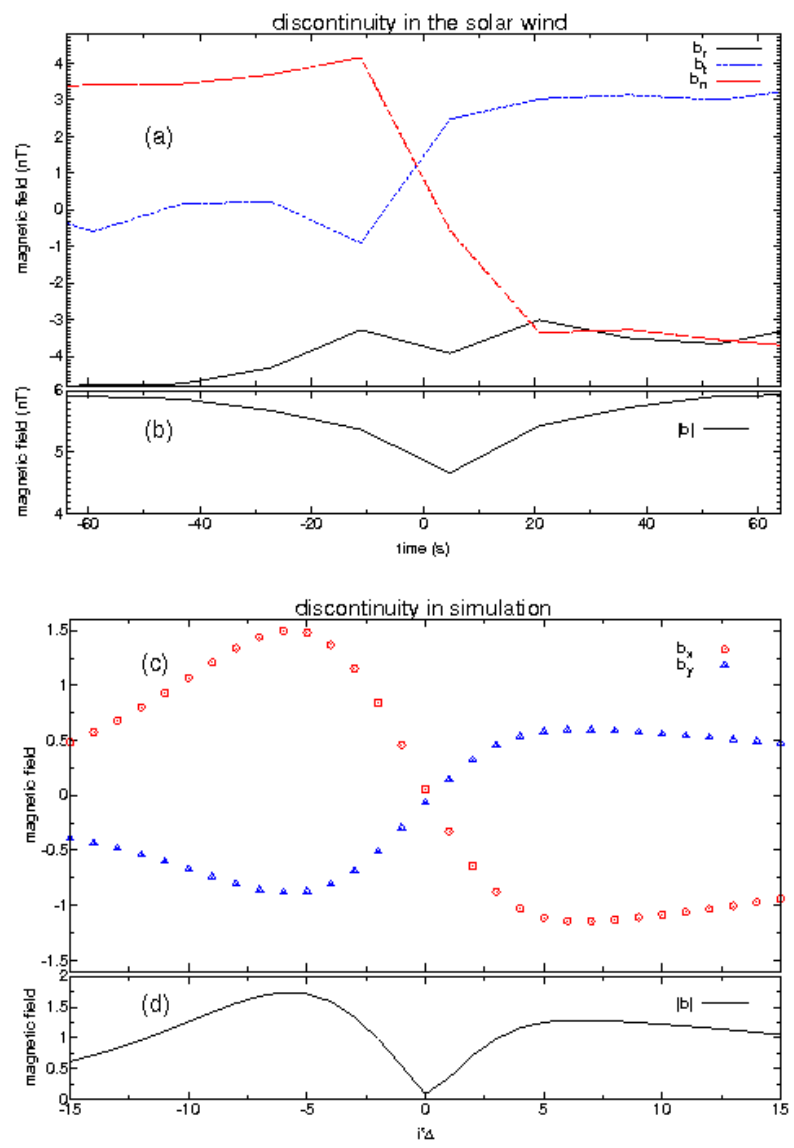

Fig. 24. Examples of discontinuities selected by the PVI method. (a): the three components of the magnetic field vector in solar wind data in the RTN reference frame; (b): magnitude of the magnetic field vector in solar wind data. The discontinuity, centered around zero, lasts few tens seconds. (c): the two components of the magnetic field vector in simulation data; (d): magnitude of the magnetic field vector in simulation data. $\Delta$ is the resolution data.

For high $\theta$, all the TDs correspond to reconnection sites. Once each reconnection site has been identified, the characteristic width $\delta^{\prime}$ can be measured, as described by Servidio et al. (2011). For each TD captured by $\Im$, we measured each $\delta^{\prime}$, and taking the average we obtained $\left\langle\delta^{\prime}\right\rangle=1.45 \times 10^{-2}$. From the 2-D simulation, as reported in Table 2, the average diffusion region thickness is $\langle\delta\rangle=1.44 \times 10^{-2}$. The estimation $\left\langle\delta^{\prime}\right\rangle$ is therefore in very good agreement with the average size of the diffusion region $\langle\delta\rangle$.

Other information such as the direction or orientation of each TD can be estimated. Using the assumption that the structures are one dimensional, in fact, there is a way to determine the normal vector to the discontinuity surface if single point measurements are used, namely the minimum variance analysis (MVA) technique (Sonnerup et al., 1967; Perri et al., 2009). We will now test this technique, making use of the fact that we have a fully 2-D picture of each RS from the

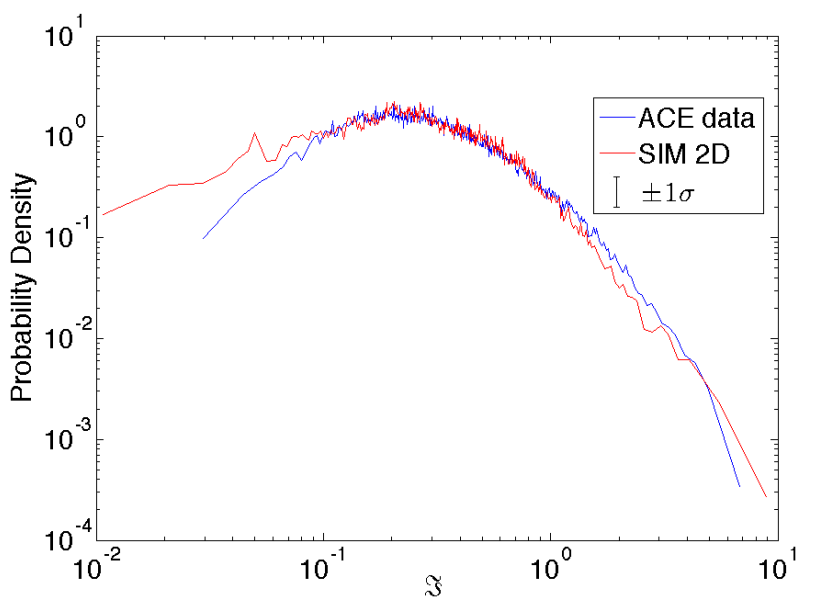

Fig. 25. Probability density function of the spatial signal $\Im$ (PVI) obtained from ACE measurements (blue line) and simulation (red line). The error bar $\pm \sigma$ is displayed in the legend and the value of $\sigma$ is the expected fractional error in the PDF due to counting statistics.

simulation (see Fig. 21). In each TD detected with the PVI and expanded using the $W$-field, we compute the matrix

$S_{i j}=\left\langle b_{i} b_{j}\right\rangle-\left\langle b_{i}\right\rangle\left\langle b_{j}\right\rangle$,

where here $\langle\ldots\rangle$ denotes an average on the trajectory within the TD. Then we compute the eigenvalues $\left(\lambda_{1}, \lambda_{2}\right)$ and the normalized eigenvectors $(\hat{\mathbf{n}}, \hat{\mathbf{t}})$, where $\lambda_{1}$ is the maximum eigenvalue and $\hat{\mathbf{n}}(\hat{\mathbf{t}})$ is the normal (tangential) eigenvector. The system of eigenvectors is shown in Fig. 23. The values of the ratio $\lambda_{1} / \lambda_{2}$ is very large for all the discontinuities selected by $\Im_{8}$, that is $100<\lambda_{1} / \lambda_{2}<10^{7}$. Another feature is that the normal component $b_{\mathrm{n}}$ is almost null and constant, while $b_{\mathrm{t}}$ is strongly changing sign (note the analogy with Eq. (8).

We have computed the PVI time series using ACE 1 second resolution magnetic field data from the interval 1 to 18 May 2004 (Osman et al., 2011). The increment $(\Delta s)$ is 20 seconds and the averaging interval in the denominator in Eq. (16) is the entire data period. The average velocity was around $400 \mathrm{~km} \mathrm{~s}^{-1}$. In Fig. 22, the PVI time series is shown. In order to facilitate the comparison, we converted the time signal to a spatial signal, using the average velocity of the flow, and then normalized to a solar wind magnetic correlation length of $1.2 \times 10^{6} \mathrm{~km}$. Imposing a threshold $\theta>8$ on Eq. (16), 704 events are identified. One of these TDs is illustrated in Fig. 24 along with an example of TD from the 2-D MHD simulation. Finally, in Fig. 25, we show the probability distribution functions of the PVI signal for both the observational and simulation data. The comparison tells us that there is a great similarity within the errors. In general, we suggest that the methods developed here may have many applications to the the solar wind data, where the coexistence of turbulence and magnetic reconnection cannot be discarded. 


\section{Conclusions}

In this paper we have assembled a digest of recent work that has examined magnetic reconnection, not as an isolated process that occurs in idealized, controlled conditions, but as a necessary ingredient in the complex nonlinear dynamical process that we call turbulence. Most, but not all, recent progress in this areas has been in the context of reduced dimensionality models that have an ignorable coordinate (i.e., 2-D or 2.5-D models.) Much of the progress in three dimensional (3-D) non-steady or turbulent reconnection has been either experimental (e.g., Ren et al., 2005; Brown et al., 2006) or in a 3-D numerical setup that is in effect nearly 2-D (Kowal et al., 2009; Daughton et al., 2011). It is noteworthy that the fully 3-D case is substantial more complex and less understood, both theoretically (Schindler et al., 1988; Priest and Pontin, 2009), and in numerics (Dmitruk and Matthaeus, 2006; Borgogno et al., 2005). However, for weakly 3-D setups, it has been amply confirmed that turbulence effects (Matthaeus and Lamkin, 1986; Servidio et al., 2009) persist (Rappazzo et al., 2007; Daughton et al., 2011). While important aspects of the physics of reconnection revealed in the 2-D paradigm can carry over to $3-\mathrm{D}$, it is likely also that there are essential physical effects that occur only in a strongly 3D system or with kinetic effects at the small scales. These challenging problems have not been included in the present discussion. Here we have concentrated on the progress in understanding reconnection in a turbulent environment that has been enabled by remaining in the relatively simple context of strong 2-D turbulence.

Most of the progress reviewed here has been in the context of nonlinear dynamics of magnetic reconnection in turbulence, investigated through direct numerical simulations of decaying 2-D MHD. In the high resolution simulations considered here, many reconnection events are seen, involving simultaneously many magnetic islands of various size. The reconnection is spontaneous but locally driven by the fields and boundary conditions provided by the turbulence. Matching classical turbulence analysis with the Sweet-Parker theory, the statistical features of these multi-scale reconnection events have been identified. Because of the complex magnetic topology, turbulence leads to different kinds of reconnecting patches that experience widely varying influences from surrounding fluctuations. This induces a great deal of variation in the observed reconnection events.

The turbulent cascade produces a distribution of reconnecting islands. Computing the electric field at the $X$-points, we see that turbulence produces a broad range of reconnection rates, with values in excess of 0.1 to 0.3 in dimensionless global Alfvén units. In addition, the strongest reconnection rates vary in proportion to $\ell / \delta$, the aspect ratio of the reconnection sites. This scaling appears superficially to differ greatly from classical laminar theories (Sweet, 1958; Parker, 1957), but taking into account the nearby magnetic field produced by the turbulence, a generalized form of Sweet-Parker scaling (Cassak and Shay, 2007) is restored. These results explain how rapid reconnection occurs in MHD turbulence in association with the most intermittent non-Gaussian current structures, and also how turbulence generates a very large number of reconnection sites that have very small rates. Reconnection, like other transport processes, is greatly affected by turbulence (Orszag, 1977) and reconnection rates, like other turbulence parameters, have a broad distribution of values.

In contrast to laminar reconnection models that provide a single predicted reconnection rate for the system, turbulent resistive MHD gives rise to a broad range of reconnection rates that depend on local turbulence parameters. Many potential reconnection sites are present, but only a few are selected by the turbulence, at a given time, to display robust reconnection electric fields. In this way, the present problem differs greatly from studies of reconnection that assume that it occurs in isolation or as a spontaneous process. In those cases, the total electric field is due to reconnection, and in steady-state, the electric field far from the diffusion region takes on the value of the reconnection electric field. Here the inductive electric fields at points removed from the $X$ points are typically larger than the reconnection rates (Servidio et al., 2010a). It seems to be appropriate therefore to view reconnection in turbulence as driven by the convective electric fields in the turbulent medium. It is this driving by the turbulent electric field that is responsible for the local flux pile-up that drives the reconnection discussed here. Flux pile-up would normally be viewed as occurring when the rate of approach of the islands is greater than the rate that can be supported by the reconnection process. This can cause buildup of repulsive forces and eventual bouncing (Intrator et al., 2009). This will be an interesting feature to study in the turbulence context but such a time-dependent correlation analysis is beyond the scope of the present work and deferred to future study.

We have seen that reconnection becomes an integral part of turbulence, as suggested previously (Matthaeus and Montgomery, 1980; Carbone et al., 1990). In fact, results of the present type may shed light on possible scalings as Reynolds numbers are increased, even though direct computational scalings remain greatly challenging. In particular, we expect that the distribution of reconnection rates can be related to the issue of maintaining finite energy dissipation in the infinite Reynolds numbers. A detailed examination of this connection remains for future study.

Very recent results in this line of study, involving reconnection rates for turbulence at varying times, and the first look at how Hall effect influences reconnection in turbulence, have also been highlighted here. From the freely decaying turbulence, time dependent study, we have found that the reconnection rate distribution evolves rapidly from a state that has essentially no fast reconnection sites, and develops a "hard" distribution that has a highly enhanced tail of strong rates, in a time of the order of the peak turbulence dissipation 
time scale. Subsequently, as the turbulence ages and begins to slow down, so also does the reconnection rate distribution soften, with the tail of strong rates diminishing in just a few nonlinear times.

The preliminary results on the influence of the Hall effect in Ohm's law on the distribution of reconnection rates in turbulence appear to be very intriguing. We find that as the Hall parameter (ratio of ion inertial length to energy containing length) is increased from zero to $1 / 100$, the distribution of reconnection rates develops a more pronounced tail at the highest values. Meanwhile the median rate is increased by only a few tens of percent. This is consistent with the idea that Hall effect can influence the fastest rates of reconnection most effectively. However many "slow" reconnection rates sites are found both with and without Hall effect. Further study will be required to confirm and complete an understanding of how Hall effect influences reconnection rates in turbulence, but the present results provide some tantalizing motivation for this future study.

The new perspective on reconnection in turbulence that we have reviewed here seems to be potentially very relevant to space and astrophysical applications such as the turbulent magnetosheath (Retinò et al., 2007), the solar wind (Gosling and Szabo, 2008; Sundkvist et al., 2007) and the solar corona (Parker, 1983). On the basis of the current results, we would expect to find in the turbulent corona and solar wind a broad distribution of size of interacting islands, with a concomitantly broad distribution of reconnection rates. The rates can, in principle, be determined statistically in terms of measurable correlation, Taylor and dissipation scales. In future studies it may be useful to study additional signatures such as characteristic reconnection flows (Gosling and Szabo, 2008). Furthermore a useful extension will be to employ models that are suited to low collisionality plasmas, where for example anomalous resistivity, or kinetic effects, may be important.

Acknowledgements. This research is partially supported by the European Project FP7-PEOPLE-2010-IRSES 269297 "TURBOPLASMAS"; the NASA Heliophysics Theory Program (NNX08AI47G); the MMS project (NNX08AT76G); the NASA Solar Probe Plus ISIS team; the NASA Geospace Sciences (NNX08AM48G), and the NSF SHINE program (ATM-0752135). S. S. would like to acknowledge the grant "Giovani Ricercatori2010" at UNICAL.

Edited by: G. Lapenta

Reviewed by: Q. Lu and M. Goldstein

\section{References}

Alexandrova, O., Carbone, V., Veltri, P., and Sorriso-Valvo, L.: Solar wind Cluster observations: Turbulent spectrum and role of Hall effect, Planet. Space Sci., 55, 2224-2227, doi:10.1016/j.pss.2007.05.022, 2007.

Birn, J., Drake, J. F., Shay, M. A., Rogers, B. N., Denton, R. E., Hesse, M., Kuznetsova, M., Ma, Z. W., Bhattacharjee, A., Otto, A., and Pritchett, P. L.: Geospace environmental modeling (GEM) magnetic reconnection, J. Geophys. Res., 106, 37153719, doi:10.1029/1999JA900449, 2001.

Biskamp, D.: Nonlinear Magnetohydrodynamics, Cambridge U. Press, Cambridge, England, 1993.

Bogdan, T. J.: The turbulent twisted magnetic flux tube gas, Phys. Fluids, 27, 994-1004, doi:10.1063/1.864690, 1984.

Borgogno, D., Grasso, D., Porcelli, F., Califano, F., Pegoraro, F., and Farina, D.: Aspects of three-dimensional magnetic reconnection, Phys. Plasmas, 12, 032309, doi:10.1063/1.1857912, 2005.

Borovsky, J.: Eddy viscosity and flow properties of the solar wind: Co-rotating interaction regions, coronal-mass-ejection sheaths, and solar-wind/magnetosphere coupling, Phys. Plasmas, 13, 056505, doi:10.1063/1.2200308, 2006.

Borovsky, J.: Flux tube texture of the solar wind: Strands of the magnetic carpet at 1 AU?, J. Geophys. Res., 113, 08110, doi:10.1029/2007JA012684, 2008.

Borovsky, J. and Denton, M. H.: Solar wind turbulence and shear: A superposed-epoch analysis of corotating interaction regions at 1 AU, J. Geophys. Res., 115, 10101, doi:10.1029/2009JA014966, 2010.

Brown, M. R., Cothran, C. D., and Fung, J.: Two fluid effects on three-dimensional reconnection in the Swarthmore Spheromak Experiment with comparisons to space data, Phys. Plasmas, 13, 056503, doi:10.1063/1.2180729, 2006.

Bruno, R. and Carbone, V.: Living Rev. Solar Phys., 2, 4, 2005.

Bruno, R., Carbone, V., Veltri, P., Pietropaolo, E., and Bavassano, B.: Identifying intermittency events in the solar wind, Planet. Space Sci., 49, 1201-1210, doi:10.1016/S0032-0633(01)000617, 2001.

Burlaga, L. F.: Micro-Scale Structures in the Interplanetary Medium, Solar Phys., 4, 67-92, doi:10.1007/BF00146999, 1968.

Burlaga, L. F. : Directional Discontinuities in the Interplanetary Magnetic Field, Solar Phys., 7, 54-71, doi:10.1007/BF00148406, 1969.

Canuto, C., Hussaini, M. Y., Quarteroni, A., and Zang, T. A.: Spectral Methods in Fluid Mechanics, Springer-Verlag, New York, 1988.

Carbone, V., Veltri, P., and Mageney, A.: Coherent structure formation and magnetic field line reconnection in magnetohydrodynamic turbulence, Phys. Fluids A, 2, 1487-1496, doi:10.1063/1.857598, 1990.

Cassak, P. A. and Shay, M. A.: Scaling of asymmetric magnetic reconnection: General theory and collisional simulations, Phys. Plasmas, 14, 102114, doi:10.1063/1.2795630, 2007.

Cassak, P. A., Shay, M. A., and Drake, J. F.: Catastrophe Model for Fast Magnetic Reconnection Onset, Phys. Rev. Lett., 235002, doi:10.1103/PhysRevLett.95.235002, 2005.

Cassak, P. A., Drake, J. F., and Eckhardt, B.: Onset of Fast Magnetic Reconnection, Phys. Rev. Lett., 98, 215001, doi:10.1088/0004637X/739/2/72, 2007.

Craig, J. D. and Watson, P. G.,: Dynamic Planar Magnetic Re- 
connection Solutions for Incompressible Plasmas, Astrophys. J., 516, 924, doi:10.1086/307135, 1999.

Daughton, W., Roytershteyn, V., Albright, B. J., Karimabadi, H., Yin, L., and Bowers, K. J.: Transition from collisional to kinetic regimes in large-scale reconnection layers, Phys. Rev. Lett., 103, 065004, doi:10.1103/PhysRevLett.103.065004, 2009.

Daughton, W., Roytershteyn, V., Karimabadi, H., Yin, L., Albright, B. J., Bergen, B., and Bowers, K. J.: Role of electron physics in the development of turbulent magnetic reconnection in collisionless plasmas, Nature Phys., 7, 539-542, doi:10.1038/nphys1965, 2011.

Dmitruk, P. and Matthaeus, W. H.: Structure of the electromagnetic field in three-dimensional Hall magnetohydrodynamic turbulence, Phys. Plasmas, 13, 042307, doi:10.1063/1.2192757, 2006.

Dmitruk, P., Matthaeus, W. H., Seenu, N., Brown, M. R.: Test Particle Acceleration in Three-dimensional Magnetohydrodynamic Turbulence, Astrophys. J., 597, L81-L84, doi:10.1086/379751, 2003.

Donzis, D. A., Yeung, P. K., and Sreenivasan, K. R.: Dissipation and enstrophy in isotropic turbulence: Resolution effects and scaling in direct numerical simulations, Phys. Fluids, 20, 045108, doi:10.1063/1.2907227, 2008.

Dorelli, J. C. and Birn, J.: Whistler-mediated magnetic reconnection in large systems: Magnetic flux pileup and the formation of thin current sheets, J. Geophys. Res., 108, 1183, doi:10.1029/2001JA009180, 2003.

Drake, J. F., Swisdak, M., Che, H., and Shay, M. A.: Electron acceleration from contracting magnetic islands during reconnection, Nature, 443, 553-556, doi:10.1038/nature05116, 2006.

Dungey, J. W.: Cosmic Electrodynamics, Cambridge University Press, England, 1958.

Fu, X. R., Lu, Q. M., and Wang, S.: The process of electron acceleration during collisionless magnetic reconnection, Phys. Plasmas, 13, 012309, doi:10.1063/1.2164808, 2006.

Galtier, S. and Buchlin, E.: Multiscale Hall-Magnetohydrodynamic Turbulence in the Solar Wind, Astrophys. J., 656, 560-566, doi:10.1086/510423, 2007.

Ghosh, S., Hossain, M., and Matthaeus, W. H.: The application of spectral methods in simulating compressible fluid and magnetofluid turbulence, Comput. Phys. Commun., 74, 18-40, doi:10.1016/0010-4655(93)90103-J, 1993.

Gosling, J. T. and Szabo, A.: Bifurcated current sheets produced by magnetic reconnection in the solar wind, J. Geophys. Res., 113, 113, A10103, doi:10.1029/2008JA013473, 2008.

Greco, A., Chuychai, P., Matthaeus, W. H., Servidio, S., and Dmitruk, P.: Intermittent MHD structures and classical discontinuities, Geophys. Res. Lett., 35, L19111, doi:10.1029/2008GL035454, 2008.

Greco, A., Matthaeus, W. H., Servidio, S., Chuychai, P., and Dmitruk, P.: Statistical Analysis of Discontinuities in Solar Wind ACE Data and Comparison with Intermittent MHD Turbulence, Astrophys. J., 691, L111, doi:10.1088/0004-637X/691/2/L111, 2009.

Hada, T., Koga, D., and Yamamoto, E.: Phase coherence of MHD waves in the solar wind, Space Sci. Rev., 107, 463-466, doi:10.1023/A:1025506124402, 2003.

Huang, C., Lu, Q., and Wang, S.: The mechanisms of electron acceleration in antiparallel and guide field magnetic reconnection,
Phys. Plasmas, 17, 072306, doi:10.1063/1.3457930, 2010.

Intrator, T. P., Sun, X., Lapenta, G., Dorf, L., and Furno, I.: Experimental onset threshold and magnetic pressure pile-up for 3-D reconnection, Nature Phys., 5, 521-526, doi:10.1038/nphys1300, 2009.

Jokipii, J. R. and Lee, M. A.: Compression Acceleration in Astrophysical Plasmas and the Production of $f(v) \propto v^{-5}$ Spectra in the Heliosphere, Astrophys. J., 713, 475-483, doi:10.1088/0004637X/713/1/475, 2010.

Koga, D. and Hada, T.: Phase coherence of foreshock MHD waves: wavelet analysis, Space Sci. Rev., 107, 495-498, doi:10.1023/A:1025510225311, 2003.

Koga, D., Chian, A. C.-L., Miranda, R. A., \& Rempel, E. L.: Intermittent nature of solar wind turbulence near the Earth's bow shock: Phase coherence and non-Gaussianity, Phys. Rev. E, 75, 046401, doi:10.1103/PhysRevE.75.046401, 2007.

Kowal, G., Lazarian, A., Vishniac, E. T., and Otmianowska-Mazur, K.: Numerical Tests of Fast Reconnection in Weakly Stochastic Magnetic Fields, Astrophys. J., 700, 63-85, doi:10.1088/0004637X/700/1/63, 2009.

Lapenta, G.: Self-Feeding Turbulent Magnetic Reconnection on Macroscopic Scales, Phys. Rev. Lett., 100, 235001, doi:10.1103/PhysRevLett.100.235001, 2008.

Lazarian, A. and Vishniac, E.: Reconnection in a Weakly Stochastic Field, Astrophys. J., 517, 700-718, doi:10.1086/307233, 1999.

Leamon, R. J., Matthaeus, W. H., Smith, C. W., Zank, G. P., Mullan, D. J., and Oughton, S.: MHD-driven Kinetic Dissipation in the Solar Wind and Corona, Astrophys. J., 537, 1054-1062, doi:10.1086/309059, 2000.

Lu, Q., Huang, C., Xie, J., Wang, R., Wu, M., Vaivads, A., and Wang, S.: Features of separatrix regions in magnetic reconnection: Comparison of 2-D particle-in-cell simulations and Cluster observations, J. Geophys. Res., 115, A11208, doi:10.1029/2010JA015713, 2010.

Ma, Z. W. and Bhattacharjee, A.: Hall magnetohydrodynamic reconnection: The Geospace Environment Modeling challenge, J. Geophys. Res., 106, 3773-3782, doi:10.1029/1999JA001004, 2001.

Malara, F., Veltri, P., and Carbone, V.: Competition among nonlinear effects in tearing instability saturation, Phys. Fluids B, 4, 3070, doi:10.1063/1.860477, 1992.

Matthaeus, W. H. and Lamkin, S. L.: Turbulent magnetic reconnection, Phys. Fluids, 29, 2513-2534, doi:10.1063/1.866004, 1986.

Matthaeus, W. H. and Montgomery, D.: Selective decay hypothesis at high mechanical and magnetic Reynolds numbers, Ann. N.Y. Acad. Sci., 357, 203-222, doi:10.1111/j.17496632.1980.tb29687.x, 1980.

Matthaeus, W. H., Ambrosiano, J. J., and Goldstein, M. L.: Particle-acceleration by turbulent magnetohydrodynamic reconnection, Phys. Rev. Lett., 53, 1449-1452, doi:10.1103/PhysRevLett.53.1449, 1984.

Matthaeus, W. H., Dmitruk, P., Smith, D., Ghosh, S., and Oughton, S.: Impact of Hall effect on energy decay in magnetohydrodynamic turbulence, Geophys. Res. Lett., 30, 2104, doi:10.1029/2003GL017949, 2003.

Matthaeus, W. H., Servidio, S., and Dmitruk, P.: Comment on "Kinetic Simulations of Magnetized Turbulence in Astrophysical Plasmas", Phys. Rev. Lett., 101, 149501, doi:10.1103/PhysRevLett.101.149501, 2008. 
Mininni, P. D. and Pouquet, A.: Finite dissipation and intermittency in magnetohydrodynamics, Phys. Rev. E, 80, 025401, doi:10.1103/PhysRevE.80.025401, 2009.

Mininni, P. D., Alexakis, A., and Pouquet, A.: Energy transfer in Hall-MHD turbulence: cascades, backscatter, and dynamo action, J. Plasma Phys, 73, 377-401, doi:10.1017/S0022377806004624, 2007.

Moffatt, H. K.: Magnetic field generation in electrically conducting fluids, Cambridge U. Press, Cambridge, England, 1978.

Montgomery, D., Matthaeus, W. H., Stribling, W. T., Martinez, D., and Oughton, S.: Relaxation in two dimensions and the 'sinh-Poisson' equation, Phys. Fluids A, 4 , 3-6, doi:10.1063/1.858525, 1992.

Mozer, F. S. and Hull, A.: Scaling the energy conversion rate from magnetic field reconnection to different bodies, Phys. Plasmas, 17, 102906, doi:10.1063/1.3504224, 2010.

Mozer, F. S. and Pritchett, P. L.: Regions associated with electron physics in asymmetric magnetic field reconnection, Geophys. Res. Lett., 36, L07102, doi:10.1029/2009GL037463, 2009.

Ness, N. F. and Burlaga, L. F.: Spacecraft studies of the interplanetary magnetic field, J. Geophys. Res., 106, 803-817, 15803, doi:10.1029/2000JA000118, 2001.

Neugebauer, M.: Comment on the abundances of rotational and tangential discontinuities in the solar wind, J. Geophys. Res., 111, A04103, doi:10.1029/2005JA011497, 2006.

Orszag, S. A.: Fluid Dynamics, edited by: Ballian, R. and Puebe, J.-L., Gordon \& Breach, NY, 235-265, 1977.

Osman, K. T., Matthaeus, W. H., Greco, A., and Servidio, S.: Evidence for Inhomogeneous Heating in the Solar Wind, Astrophys. J., 727, L11, doi:10.1088/2041-8205/727/1/L11, 2011.

Parker, E. N.: Sweet's Mechanism for Merging Magnetic Fields in Conducting Fluids, J. Geophys. Res., 62, 509-520, doi:10.1029/JZ062i004p00509, 1957.

Parker, E. N.: Magnetic neutral sheets in evolving fields. Formation of the solar corona, Astrophys. J., 62, 509-520, doi:10.1029/JZ062i004p00509, 1957.

Paschmann, G.: Recent in-situ observations of magnetic reconnection in near-Earth space, Geophys. Res. Lett., 35, Issue 19, L19109, doi:10.1029/2008GL035297, 2008.

Patterson, G. S. and Orszag, S. A.: Spectral Calculations of Isotropic Turbulence: Efficient Removal of Aliasing Interactions, Phys. Fluids, 14, 2538-2541, doi:10.1063/1.1693365, 1971.

Perri, S., Yordanova, E., Carbone, V., Veltri, P. , Sorriso-Valvo, L., Bruno, R., and André, M.: Magnetic turbulence in space plasmas: Scale-dependent effects of anisotropy, J. Geophys. Res., 114, A02102, doi:10.1029/2008JA013491, 2009.

Phan, T. D., Gosling, J. T., Paschmann, G., Pasma, C., Drake, J. F., Oieroset, M., Larson, D., Lin, R. P., and Davis, M. S. : The Dependence of Magnetic Reconnection on Plasma and Magnetic Shear: Evidence from Solar Wind Observations, Astrophys. J., 719, L199-L203, doi:10.1088/2041-8205/719/2/L199, 2010.

Priest, E. R. and Pontin, D. I.: Three-dimensional null point reconnection regimes, Phys. Plasmas, 16, 122101, doi:10.1063/1.3257901, 2009.

Pritchett, P. L. and Mozer, F. S.: Asymmetric magnetic reconnection in the presence of a guide field, J. Geophys. Res., 114, A11210, doi:10.1029/2009JA014343, 2009.

Rana, S.: Surface Topological Data Structures: An Introduction for Geographical Information Science, John Wiley and Sons, Chich- ester, England, doi:10.1002/0470020288, 2004.

Rappazzo, A. F., Velli, M., Einaudi, G., and Dahlburg, R. B., Coronal Heating, Weak MHD Turbulence, and Scaling Laws, Astrophys. J., 657, L47-L51, doi:10.1086/512975, 2007.

Ren, Y., Yamada, M., Gerhardt, S., Ji, H., Kulsrud, R, and Kuritsyn, A.: Experimental Verification of the Hall Effect during Magnetic Reconnection in a Laboratory Plasma, Phys. Rev. Lett., 95, 055003, doi:10.1103/PhysRevLett.95.055003, 2005.

Retinò, A., Sundkvist, D., Vaivads, A., Mozer, F., André, M., and Owen, C. J.: In situ evidence of magnetic reconnection in turbulent plasma, Nature Phys., 3, 235-238, doi:10.1038/nphys574, 2007.

Roberts, K. V. and Taylor, J. B.: Magnetohydrodynamic Equations for Finite Larmor Radius, Phys. Rev. Lett., 3, 197-198, doi:10.1103/PhysRevLett.8.197, 1962.

Sato, T., Hayashi, T., and Tamao, T.: Confinement and jetting of plasmas by magnetic reconnection, Phys. Rev. Lett., 41, 15481551, doi:10.1103/PhysRevLett.41.1548, 1978.

Sahraoui, F.: Diagnosis of magnetic structures and intermittency in space-plasma turbulence using the technique of surrogate data, Phys. Rev. E, 78, 026402, doi:10.1103/PhysRevE.78.026402, 2008.

Sahraoui, F. and Goldstein, M.: Structures and Intermittency in Small Scales Solar Wind Turbulence Twelfth International Solar Wind Conference, AIP Conference Proceedings, 1216, 140, 2010.

Schindler, K., Hesse, M., and Birn, J.: General magnetic reconnection, parallel electric fields, and helicity, J. Geophys. Res., 93, 5547-5557, doi:10.1029/JA093iA06p05547, 1988.

Servidio, S., Carbone, V., Primavera, L., Veltri, P., and Stasiewicz, K.: Compressible turbulence in Hall Magnetohydrodynamics, Planet. Space Sci., 55, 2239-2243, doi:10.1016/j.pss.2007.05.023, 2007.

Servidio, S., Matthaeus, W. H., and Dmitruk, P.: Depression of Nonlinearity in Decaying Isotropic MHD Turbulence, Phys. Rev. Lett., 100, 095005, doi:10.1103/PhysRevLett.100.095005, 2008.

Servidio, S., Matthaeus, W. H., Shay, M. A., Cassak, P. A., and Dmitruk, P.: Magnetic Reconnection in Two-Dimensional Magnetohydrodynamic Turbulence, Phys. Rev. Lett., 102, 115003, doi:10.1103/PhysRevLett.102.115003, 2009.

Servidio, S., Matthaeus, W. H., Shay, M. A., Dmitruk, P., Cassak, P. A., and Wan, M.: Statistics of magnetic reconnection in twodimensional magnetohydrodynamic turbulence, Phys. Plasmas, 17, 032315, doi:10.1063/1.3368798, 2010a.

Servidio, S., Wan, M., Matthaeus, W. H., and Carbone, V.: Local relaxation and maximum entropy in two-dimensional turbulence: Phys. Fluids, 22, 125107, doi:10.1063/1.3526760, 2010b.

Servidio, S., Greco, A., Matthaeus, W. H., Osman, K. T., and Dmitruk, P.: Statistical association of discontinuities and reconnection in magnetohydrodynamic turbulence, J. Geophys. Res., 116, A09102, 1-11, doi:10.1029/2011JA016569, 2011.

Smith, D., Ghosh, S., Dmitruk, P., and Matthaeus, W. H.: Hall and Turbulence Effects on Magnetic Reconnection, Geophys. Res. Lett., 31, L02805, doi:10.1029/2003GL018689, 2004.

Sonnerup, B. U. O.: Theory of magnetic reconnection in incompressible fluids, J. Plasma Phys., 4, 161-174, doi:10.1017/S0022377800004888, 1970.

Sonnerup, B. U. O. and Cahill, L. J.: Magnetopause Structure and Attitude from Explorer 12 Observations, J. Geophys. Res., 72, 
171-183, doi:10.1029/JZ072i001p00171, 1967.

Sonnerup, B. U. O., Paschmann, G., Papamastorakis, I., Sckopke, N., Haerendel, G., Bame, S. J., Asbridge, J. R., Gosling, J. T., and Russel, C. T.: Evidence for Magnetic Field Reconnection at the Earth's Magnetopause, J. Geophys. Res., 86, 10049-10067, doi:10.1029/JA086iA12p10049, 1981.

Sorriso-Valvo, L., Carbone, V., Veltri, P., Consolini, G., and Bruno, R.: Intermittency in the solar wind turbulence through probability distribution functions of fluctuations, Geoph. Res. Lett., 26, 1801-1804, doi:10.1029/1999GL900270, 1999.

Strauss, H. R.: Turbulent reconnection, Astrophys. J., 326, 412417, doi:10.1086/166104, 1988.

Sweet, P. A.: Electromagnetic Phenomena in Cosmical Physics, Cambridge University Press, New York, 1958.

Sundkvist, D., Retino, A., Vaivads A., and Bale, S. D.: Dissipation in Turbulent Plasma due to Reconnection in Thin Current Sheets, Phys. Rev. Lett., 99, 025004, doi:10.1103/PhysRevLett.99.025004, 2007.

Taylor, J. B.: Relaxation and magnetic reconnection in plasmas, Rev. Mod. Phys.,58, 741-763, doi:10.1103/RevModPhys.58.741, 1986.

Tsurutani, B. T. and Smith, E. J.: Interplanetary discontinuities Temporal variations and the radial gradient from 1 to $8.5 \mathrm{AU}, \mathrm{J}$. Geophys. Res., 84, 2773-2787, doi:10.1029/JA084iA06p02773, 1979.

Vasquez, B. J., Abramenko, V. I., Haggerty, D. K, and Smith, C. W.: Numerous small magnetic field discontinuities of Bartels rotation 2286 and the potential role of Alfvnic turbulence, J. Geophys. Res., 112, 11102, doi:10.1029/2007JA012504, 2007.
Vasyliunas, V. M.: Theoretical models of magnetic field line merging, I, Rev. Geophys. Space Phys., 13, 303-336, doi:10.1029/RG013i001p00303, 1975.

Veltri, P.: MHD turbulence in the solar wind: self-similarity, intermittency and coherent structures, Plasma Phys. Control. Fusion, 41, A787-A795, doi:10.1088/0741-3335/41/3A/071, 1999.

Veltri, P. and Mangeney, A.: Scaling laws and intermittent structures in solar wind MHD turbulence, Solar Wind IX, AIP Conference Proceedings, 471, 543, 1999.

Wan, M., Oughton, S., Servidio, S., and Matthaeus, W. H.: Generation of non-Gaussian statistics and coherent structures in ideal magnetohydrodynamics, Phys. Plasmas, 16, 080703, doi:10.1063/1.3206949, 2009.

Wan, M., Oughton, S., Servidio, S., and Matthaeus, W. H.: On the accuracy of simulations of turbulence, Phys. Plasmas, 17, 082308, doi:10.1063/1.3474957, 2010.

Wang, R., Lu, Q., Du, A., and Wang, S.: In Situ Observations of a Secondary Magnetic Island in an Ion Diffusion Region and Associated Energetic Electrons, Phys. Rev. Lett., 104, 175003, doi:10.1103/PhysRevLett.104.175003, 2010.

Webb, G. M., Ko, C. M., Zank, G. P., and Jokipii, J. R.: DiffusiveCompression Acceleration and Turbulent Diffusion of Cosmic Rays in Quasi-periodic and Turbulent Flows, Astrophys. J., 595, 195-226, doi:10.1086/377355, 2003.

Yamada, M.: Progress in understanding magnetic reconnection in laboratory and space astrophysical plasmas, Phys. Plasmas, 14, 058102, doi:10.1063/1.2740595, 2007.

Zweibel, E. G. and Rhoads, J. E. : Magnetic merging in colliding flux tubes, Astrophys. J., 440, 407-414, doi:10.1086/175282, 1995. 